\title{
COHOMOLOGICAL STRATIFICATION OF DIAGRAM ALGEBRAS
}

\author{
ROBERT HARTMANN, ANNE HENKE, STEFFEN KOENIG, ROWENA PAGET
}

\section{INTRODUCTION}

In 1901 Issai Schur proved a fundamental result, which in modern language reads as follows: Let $k=\mathbb{C}$ be the field of complex numbers, $n$ and $r$ integers, $\Sigma_{r}$ the symmetric group on $r$ letters, $G=G L_{n}(k)$ the general linear group of invertible $n$ by $n$ matrices, and $\mathrm{Pol}_{r}$ the category of polynomial representations of $G$ that are homogeneous of degree $r$ over $\mathbb{C}$. Then $\mathrm{Pol}_{r}$ is equivalent to the subcategory of $\mathbb{C} \Sigma_{r}$-mod of modules having composition factors indexed by partitions of $r$ into not more than $n$ parts. In particular, if $n \geq r$ then $\mathrm{Pol}_{r}$ is equivalent to $\mathbb{C} \Sigma_{r}$-mod.

What happens if we replace $k$ by an algebraically closed field of prime characteristic? In this case, Schur's result appears to be completely wrong. Not only is Schur's functor far away from being an equivalence. Even worse, the category $\mathrm{Pol}_{r}$ always has finite global dimension, while $k \Sigma_{r}$-mod and its relevant subcategories usually have cohomology in infinitely many degrees. Thus no exact categorical equivalence is possible. However, modular representation theory suggests considering a more sophisticated setup when trying to extend Schur's theorem. Indeed, the simple representations in characteristic zero of either $G$ or $\Sigma_{r}$ have integral versions. These lattices can be reduced to prime characteristic, providing (in general non-simple) modular analogues of the characteristic zero simples. Well-known analogues are the Weyl modules $\Delta(\lambda)$ in the case of the general linear group, and the cell modules $S(\lambda)$ in the case of the symmetric group (here taken to be the dual Specht modules); in either case, the indices $\lambda$ are partitions. Hence the modular reduction process suggests considering a relative version of Schur's result: $\mathcal{F}(\Delta) \simeq \mathcal{F}(S)$ - an equivalence between a category of $G$-modules with a Weyl filtration and a category of $k \Sigma_{r}$-modules with a cell filtration. This, however, is still wrong, as shown by small examples such as $\Sigma_{r}$ with $r=2,3$ in characteristic two or three.

It came as a major surprise when Hemmer and Nakano [13] recently proved, using results on cohomology of symmetric groups, that in prime characteristic Schur's theorem is almost relatively true. That is, the above relative version $\mathcal{F}(\Delta) \simeq \mathcal{F}(S)$ of Schur's result is true provided one excludes characteristic two or three. In other words, the category of cell filtered representations of symmetric groups behaves (mostly) like a subcategory of a highest weight category in algebraic Lie theory. In more technical terms, this means there is a partial order on the indices $\lambda$ such that $\operatorname{Hom}(S(\lambda), S(\mu)) \neq 0 \mathrm{im}$ plies $\lambda \leq \mu$ and $\operatorname{Ext}^{1}(S(\lambda), S(\mu)) \neq 0$ even implies $\lambda<\mu$, which is rather unexpected from the point of view of representations of finite groups. The result by Hemmer and 
Nakano exhibits a new phenomenon, which is however not isolated. Indeed a recent result [12] by Hartmann and Paget establishes the same phenomenon for Brauer algebras - with a similar set of cases to be excluded.

We are going to establish the Hemmer-Nakano phenomenon for a large class of algebras. We mostly work with an axiomatically defined class of algebras, see Definition 2.1. The main feature of this definition is, however, that it includes many known classes of socalled diagram algebras, which have been used extensively in knot theory, $C^{*}$-algebras, mathematical physics or representation theory for various reasons. In particular, we will cover Brauer algebras (thus reproving results of [12]), Birman-Wenzl-Murakami (BMW) algebras and partition algebras. In all cases we will find almost relatively true versions of Schur's theorem, see Theorem 10.2 and Corollary 10.3.

While diagram algebras are usually defined in combinatorial terms, the main techniques employed here are of a categorical and cohomological nature. In particular, we will provide stratifications of derived module categories of the algebras we study, see Proposition 7.2 and Theorem 7.3. We will relate these stratifications to the cellular structures of our algebras, which in our examples are so-called iterated inflations of various symmetric groups or their Hecke algebras. On a technical level, we actually will find two kinds of stratifications. One of them is on the level of derived categories, and this stratification is a new feature of our algebras, not visible for group algebras of symmetric groups. The second (relative) stratification is the analogue of the HemmerNakano result.

Apart from the results mentioned above, the methods employed also yield a variety of new results on the diagram algebras studied, and the methods and results are likely to carry over to other diagram algebras too. On a numerical level we will identify many unknown decomposition numbers of the algebras studied with known or unknown decomposition numbers of symmetric groups, see Proposition 6.2. Moreover, cell filtration multiplicities are well-defined, see Theorem 10.2 (b). On a structural level, we get comparisons of cohomology which for instance allow us to apply known results of the cohomology of symmetric groups to diagram algebras. Moreover, there are several vanishing results for extensions between cell modules, see Sections 7 and 8 . Furthermore, we will verify the finitistic dimension conjecture for the algebras studied, which is known to imply various other conjectures, see Corollary 7.6. Algebras such as the Brauer algebra occur in representation theory of symplectic or orthogonal groups. Thus, all of these comparisons, on a numerical or structural level, have the feature of relating types $\mathrm{B}$ or $\mathrm{C}$ with type $\mathrm{A}$ (symmetric groups).

In a wider context, the results of this article also have the following features: The algebras we are going to study are cellular and usually not quasi-hereditary, thus they do not fall into the customary setup of algebraic Lie theory. However, our methods yield the existence of Schur algebras for our algebras, and these Schur algebras have the usual features known for Schur algebras of classical groups or blocks of the BernsteinGelfand-Gelfand category of a semisimple complex Lie algebra. In particular, these new - and yet to be studied - Schur algebras are quasi-hereditary, that is, their module categories are highest weight categories. These new Schur algebras are in Schur-Weyl duality with the diagram algebras studied in this article, see Theorem 13.1. We also 
can keep various features of symmetric groups such as the existence of Young modules, which in the case of the symmetric groups are direct summands of permutation modules. Results such as Theorem 13.1 can be seen as providing a hidden algebraic Lie theory for the diagram algebras we are studying.

As mentioned, the diagram algebras we are studying are cellular algebras, but usually not quasi-hereditary. Cellular algebras keep some of the numerical features of quasihereditary algebras, while structural features are usually lost when passing from quasihereditary to cellular algebras. Another generalization of quasi-hereditary algebras, stratified algebras, has been defined and studied with the aim of keeping structural properties such as stratifications of derived categories. All three classes of algebras are defined by the existence of certain chains of ideals. Formally, a cell ideal that is stratifying must be heredity and a stratifying ideal that is cell must be heredity, too. That is, in a formal sense, the intersection of cellular and stratified is quasi-hereditary. However, the diagram algebras studied here, and the axiomatically defined class of cellularly stratified algebras we are going to study, combine cellular and stratified features in a new way. This new generalization of quasi-hereditary algebras appears to preserve or to extend naturally the fundamental properties of quasi-hereditary algebras, and to connect the two theories of cellular and stratified algebras and their rather different sets of methods.

This article is organized as follows: In Section 2 we give an axiomatic definition, phrased in combinatorial terms, of the abstract class of algebras to be studied, and then we verify that three classes of examples fit into this setup: Brauer algebras, BMW algebras and partition algebras. One feature of our algebra $A$ is to have a chain of ideals whose subquotients (layers) are related to other algebras $B$, which in the examples are group algebras of symmetric groups or deformations of those. Section 3 sets up the categorical technology to compare the module categories of $A$ and $B$. Three crucial ring theoretical and homological conditions are identified and verified, which provide the basis of the main results. In particular, this structure gives an induction functor whose properties are studied in Section 4. In Section 5 we show that our algebras can be characterised by these structural properties, this yields an alternative definiton. In Section 6, we relate some decomposition numbers of the algebra $A$ with decomposition numbers of the smaller algebras $B$. Section 7 investigates the whole chain of ideals, which turns out to yield a stratification of the derived category of $A$. Section 8 strengthens these results by proving further vanishing results for homomorphism and extension spaces. Section 9 gives a detailed example. Finally, Sections 10 to 13 use the results of Sections 3, 7 and 8 to achieve the main results, summarized in Theorems 10.2 and 13.1, including the Hemmer-Nakano phenomenon, the existence of Schur algebras, and Schur-Weyl duality.

Acknowledgement. The authors would like to thank the referee for all their very detailed comments. 


\section{Cellularly stratified algebras - Definition and examples}

The main objects studied in this article are certain cellular algebras. Cellular algebras were introduced in [9] and, subsequently, an equivalent definition was given in [17]. It was shown in [18] that every cellular algebra can be constructed by iterated inflations of smaller cellular algebras. In this section we provide an axiomatic definition of the class of cellular algebras to be studied in this article, so called cellularly stratified algebras. We then give three classes of diagram algebras which are cellularly stratified.

2.1. Cellularly stratified algebras. Let $A$ be an algebra (with identity) which can be realized as an iterated inflation of cellular algebras $B_{l}$ along vector spaces $V_{l}$ for $l=1, \ldots, n$. By $[18$, Section 3.1], this implies that as a vector space

$$
A=\bigoplus_{l=1}^{n} B_{l} \otimes V_{l} \otimes V_{l},
$$

and $A$ is cellular with a chain of two-sided ideals $\{0\}=J_{0} \subseteq J_{1} \subseteq \ldots \subseteq J_{n}=A$, which can be refined to a cell chain, and each subquotient $J_{l} / J_{l-1}$ equals $B_{l} \otimes V_{l} \otimes V_{l}$ as an algebra without unit. The involution $i$ of $A$, an anti-automorphism with $i^{2}=i d$, is defined through the involutions $j_{l}$ of the cellular algebras $B_{l}$ where

$$
i(b \otimes u \otimes v)=j_{l}(b) \otimes v \otimes u
$$

for any $b \in B_{l}$ and $u, v \in V_{l}$. Recall that the multiplication rule of a layer $B_{l} \otimes V_{l} \otimes V_{l}$ is dictated by the axioms of inflation and given by

$$
(b \otimes x \otimes y) \cdot\left(b^{\prime} \otimes x^{\prime} \otimes y^{\prime}\right)=\left(b \varphi\left(y, x^{\prime}\right) b^{\prime} \otimes x \otimes y^{\prime}\right)+\text { lower terms, }
$$

for $b, b^{\prime} \in B_{l}, x, x^{\prime}, y, y^{\prime} \in V$, where $\varphi$ is the bilinear form coming with the inflation data. Here lower terms refers to elements in lower layers $B_{h} \otimes V_{h} \otimes V_{h}$ for $h<l$. For more details on inflations see [18] and also the examples below. Let $1_{B_{l}}$ be the unit element of the algebra $B_{l}$. We define:

Definition 2.1. A finite dimensional associative algebra $A$ over a field $k$ is called cellularly stratified with stratification data $\left(B_{1}, V_{1}, \ldots, B_{n}, V_{n}\right)$ if and only if the following conditions are satisfied:

(C) The algebra $A$ is an iterated inflation of cellular algebras $B_{l}$ along vector spaces $V_{l}$ for $l=1, \ldots, n$.

(E) For each $l=1, \ldots, n$ there exist non-zero elements $u_{l}, v_{l} \in V_{l}$ such that

$$
e_{l}:=1_{B_{l}} \otimes u_{l} \otimes v_{l}
$$

is an idempotent.

(I) If $l>m$, then $e_{l} e_{m}=e_{m}=e_{m} e_{l}$.

Condition (C) implies that $A$ is cellular, see [18, Proposition 3.5]. The name 'stratified' will be justified in Section 7 , when we will show that cellularly stratified algebras are stratified in the sense of [3].

Remark. (a) By the definition of an iterated inflation (see assumption 3.4 in [18]), the top layer satisfies $V_{n}=k$, and hence $e_{n}=1$. Again by the definition of iterated inflation, the algebra $B_{n}$ is a quotient algebra of $A$. 
(b) Let $u, v \in V_{l}$ be any elements such that $1 \otimes u \otimes v$ is an idempotent. We claim that then $\varphi(v, u)=1=\varphi(u, v)$ : By the multiplication in $A$, see Equation (3), we have

$(1 \otimes u \otimes v)(1 \otimes u \otimes v)=\varphi(v, u) \otimes u \otimes v+$ lower terms.

Since $1 \otimes u \otimes v$ is an idempotent, it follows that there are no lower terms and $\varphi(v, u)=1$. Using the involution, we have $1_{B_{l}}=j_{l}\left(1_{B_{l}}\right)$ and hence $i\left(1_{B_{l}} \otimes u \otimes v\right)=1_{B_{l}} \otimes v \otimes u$. Doing the same calculation as in (4) for $i\left(1_{B_{l}} \otimes u \otimes v\right)$, it also follows that $\varphi(u, v)=1$.

Lemma 2.2. Let $A$ be cellularly stratified and $1 \leq l \leq n$. The following holds:

(1) The ideal $J_{l}$ is generated by $e_{l}$, that is, $J_{l}=A e_{l} A$.

(2) The algebra $A / J_{l}$ is cellularly stratified.

Proof. Assume we are in the lowest layer, that is $l=1$. Then for any $x, y \in V_{1}$ and $b \in B_{1}$ we have

$$
\left(b \otimes x \otimes v_{1}\right)\left(1 \otimes u_{1} \otimes v_{1}\right)\left(1 \otimes u_{1} \otimes y\right)=\left(b \otimes x \otimes v_{1}\right)\left(1 \otimes u_{1} \otimes y\right)=b \otimes x \otimes y
$$

as there are no lower terms and as $\varphi\left(v_{1}, u_{1}\right)=1$ by the above remark. Hence the lowest layer $J_{1}=A e_{1} A$ is generated by $e_{1}$. Assume next that the ideal $J_{l-1}$ is generated by the element $e_{l-1}$. Since $e_{l-1} e_{l}=e_{l-1}$ by assumption (I), all elements in $J_{l-1}$ can be generated by $e_{l}$. Moreover, in layer $l$ we have for any $c \in B_{l}$ and $x, y \in V_{l}$ :

$$
\left(c \otimes x \otimes v_{l}\right)\left(1 \otimes u_{l} \otimes v_{l}\right)\left(1 \otimes u_{l} \otimes y\right)=c \otimes x \otimes y+\text { lower terms. }
$$

By the above, the lower terms can be generated by $e_{l}$. Hence $c \otimes x \otimes y$ can be generated by $e_{l}$, and the first claim follows. The second claim follows from the definition of cellularly stratified.

Lemma 2.3. Let $A$ be cellularly stratified. With the set-up as in Definition 2.1, there is an algebra isomorphism $B_{l} \simeq e_{l} A e_{l} / e_{l} J_{l-1} e_{l}$ with $1_{B_{l}}$ mapped to $e_{l}$.

Proof. Since the index $l$ is fixed, it will be omitted. We will denote $J_{l-1}$ by $J^{\prime}$. As algebras, $e A e / e J^{\prime} e \simeq\left(e+J^{\prime}\right)\left(A / J^{\prime}\right)\left(e+J^{\prime}\right) \simeq\left(e+J^{\prime}\right)\left(J / J^{\prime}\right)\left(e+J^{\prime}\right)$. Using the multiplication in $A$, we see that $\left(e+J^{\prime}\right)\left(J / J^{\prime}\right)\left(e+J^{\prime}\right)$ is spanned by elements $b \otimes u \otimes v+J^{\prime}$, where $b \in B$. Using the fact that $\varphi(v, u)=1$, all such elements lie in $\left(e+J^{\prime}\right)\left(J / J^{\prime}\right)\left(e+J^{\prime}\right)$ since

$$
b \otimes u \otimes v+J^{\prime}=\left(1 \otimes u \otimes v+J^{\prime}\right)\left(b \otimes u \otimes v+J^{\prime}\right)\left(1 \otimes u \otimes v+J^{\prime}\right) .
$$

The map $B \rightarrow\left(e+J^{\prime}\right)\left(J / J^{\prime}\right)\left(e+J^{\prime}\right)$ given by $b \mapsto b \otimes u \otimes v+J^{\prime}$ is then bijective. It is a homomorphism since

$$
\left(b \otimes u \otimes v+J^{\prime}\right)\left(b^{\prime} \otimes u \otimes v+J^{\prime}\right)=b \varphi(v, u) b^{\prime} \otimes u \otimes v+J^{\prime}=b b^{\prime} \otimes u \otimes v+J^{\prime} .
$$

Next we will give examples of cellularly stratified algebras. All examples given are 'diagram algebras', meaning that they have a basis which can be represented by certain diagrams. Instead of writing down the elements $u_{l}, v_{l}$ in the following, we will give the idempotents $e_{l}$. Note that the labelling of the ideal chains is different from the one in Definition 2.1. 
2.2. Brauer algebras. Recall that for $r \in \mathbb{N}$ and $\delta \in k$, the Brauer algebra $B_{k}(r, \delta)$ has $k$-basis the set of diagrams of the following form: a diagram has $2 r$ vertices arranged in two rows of $r$ vertices, and $r$ edges such that each vertex is incident to precisely one edge. To multiply two diagrams, the diagrams are concatenated and any closed loops appearing are removed. If $c$ closed loops are removed from the concatenation to give another diagram $d$ then the product is defined to be $\delta^{c} \cdot d$. More details and an example of the multiplication can be found, for example, in [21].

Graham and Lehrer [9] showed that $B_{k}(r, \delta)$ is a cellular algebra, with the involution $i$ given by reflecting diagrams in the horizontal line cutting diagrams into an upper and a lower half. In [21], a different proof of the cellularity has been given, by showing that Brauer algebras are iterated inflations of group algebras of symmetric groups. Let us recall some details of this. We define $J_{l}$ to be the subspace of $B_{k}(r, \delta)$ with basis all diagrams with at most $l$ 'through strings', that is, edges joining a vertex in the top row of the diagram to a vertex in the bottom row. Then $J_{l}$ is a two-sided ideal of $B_{k}(r, \delta)$ and we obtain a filtration of the Brauer algebra:

$$
0 \subseteq J_{t} \subseteq J_{t+2} \subseteq \ldots \subseteq J_{r-2} \subseteq J_{r}=B_{k}(r, \delta)
$$

where $t$ is 0 or 1 depending on whether $r$ is even or odd. The subquotient $J_{l} / J_{l-2}$ is isomorphic to an inflation $k \Sigma_{l} \otimes V_{l} \otimes V_{l}$ of $k \Sigma_{l}$ along a vector space $V_{l}$ as given in [21, Lemma 5.3]. Here we define $J_{0} / J_{-2}=J_{0}$ and $J_{1} / J_{-1}=J_{1}$. This realizes $B_{k}(r, \delta)$ as an iterated inflation of group algebras of symmetric groups, see [21, Theorem 5.6]. As a free $k$-module

$$
B_{k}(r, \delta)=k \Sigma_{r} \oplus\left(k \Sigma_{r-2} \otimes V_{r-2} \otimes V_{r-2}\right) \oplus\left(k \Sigma_{r-4} \otimes V_{r-4} \otimes V_{r-4}\right) \oplus \ldots,
$$

and the iterated inflation starts with $k \Sigma_{r}$, inflates it along $k \Sigma_{r-2} \otimes V_{r-2} \otimes V_{r-2}$ and so on, ending with an inflation of $k=k \Sigma_{1}$ or $k=k \Sigma_{0}$ as bottom layer, depending on whether $r$ is odd or even. We shall see that $B_{k}(r, \delta)$ is cellularly stratified in the cases where $\delta \neq 0$ or $\delta=0$ and $r$ is odd. By [21, Theorem 5.6] assumption (C) is satisfied, since group algebras of symmetric groups are cellular $[9,(1.2)]$. For $\delta \neq 0$ and for $l=r, r-2, \ldots, t$, we choose $e_{l}$ to be

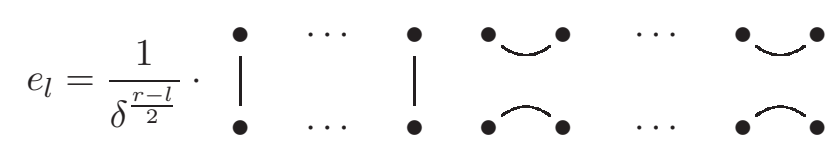

where this diagram has $l$ through strings. If $\delta=0$ and $r$ is odd then we define $e_{l}$ to be the following diagram with $l$ through strings:

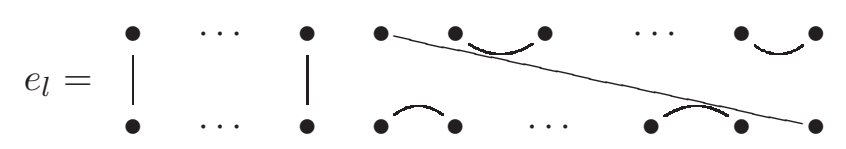

In each case the element $e_{l}$ is an idempotent of $B_{k}(r, \delta)$, so (E) holds. It easily is checked that (I) is satisfied. Observe that the condition $\delta \neq 0$ when $r$ is even is necessary since otherwise the non-zero ideal $J_{0}$ is nilpotent, and hence not generated by an idempotent. We have proved:

Proposition 2.4. Let $k$ be any field, $r$ an integer and $\delta \in k$. If $r$ is even, suppose $\delta \neq 0$. Then the Brauer algebra $B_{k}(r, \delta)$ is cellularly stratified. 
Note that $e_{l} B_{k}(r, \delta) e_{l} \subseteq B_{k}(r, \delta)$ is isomorphic to $B_{k}(l, \delta)$ and has a subalgebra isomorphic to $k \Sigma_{l}$.

Using the results of Hemmer and Nakano [13], it has been shown in [12] that, in the above cases, the cell modules of $B_{k}(r, \delta)$ form a standard system (see Section 10) if and only if the characteristic of $k$ is neither two nor three, and it was then deduced that filtration multiplicities are well-defined for $B_{k}(r, \delta)$-modules with a cell filtration ([12, Thm 2]). Results on decomposition numbers were also obtained in [12, Prop 2 and 6]. We will derive these statements again from the general set-up in this article.

2.3. Birman-Murakami-Wenzl algebras (BMW algebras). The BMW algebras are deformations of Brauer algebras. Xi [25] showed that BMW algebras are cellular, and moreover they are iterated inflations of Hecke algebras $\mathcal{H}$ of symmetric groups ([25, Section 3]). For $r \in \mathbb{N}, \lambda, \lambda^{-1}, q, q^{-1}, \delta \in k$ satisfying $\lambda^{-1}-\lambda=\left(q-q^{-1}\right)(\delta-1)$, the BMW algebra

$$
B M W_{r}:=B M W_{k}\left(r, \lambda, q-q^{-1}, \delta\right)
$$

can be defined by generators and relations (see [25]). But instead we use the equivalent definition which displays $B M W_{r}$ as a diagram algebra, with a basis of certain $r$-tangles, subject to certain relations. Given a Brauer diagram $d$ on $2 r$ vertices, one can define an $r$-tangle $T_{d}$ by a rule which specifies which strings of $d$ should cross over and which cross under. For details we refer the reader to [25, Section 2.2]. Then $\left\{T_{d}: d\right.$ a Brauer diagram $\}$ is a $k$-basis for $B M W_{r}$. Xi goes on to define $J_{l}$ to be the $k$-module generated by elements $T_{d}$ where $d$ is a Brauer diagram with at most $l$ through strings. Then:

$$
0 \subseteq J_{t} \subseteq \cdots \subseteq J_{r-2} \subseteq J_{r}=B M W_{r}
$$

is a chain of two-sided ideals where $t$ is 1 or 0 depending on whether $r$ is odd or even, and

$$
J_{l} / J_{l-2} \simeq \mathcal{H}_{l}\left(q^{-2}\right) \otimes V_{l} \otimes V_{l}
$$

for some vector space $V_{l}$ (see [25], 3.5), and where $\mathcal{H}_{l}\left(q^{-2}\right)$ is a Hecke algebra. Thus $B M W_{r}$ satisfies assumption (C). If $\delta \neq 0$ we define for each $l=r, r-2, \ldots$ the idempotent $e_{l}$ by

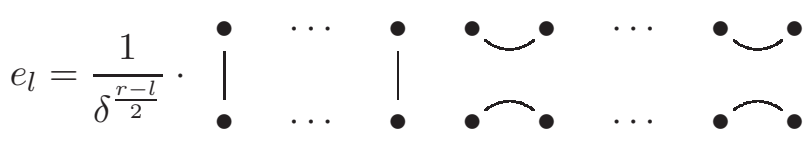

where the diagram is an $r$-tangle with $l$ vertical lines. If $\delta=0$ and $r$ is odd we define for each $l=r, r-2, \ldots, 1$ the idempotent $e_{l}$ by

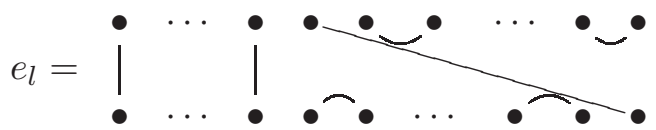

Then it is clear from the definitions that $e_{l} B M W_{r} e_{l} \simeq B M W_{l}$. We find that all the assumptions are satisfied, and we obtain that $B M W_{r}$ is cellularly stratified.

Proposition 2.5. Let $k$ be any field, $r$ an integer and $\delta \in k$. If $r$ is even, suppose $\delta \neq 0$. Then the $B M W$ algebra $B M W_{k}\left(r, \lambda, q-q^{-1}, \delta\right)$ is cellularly stratified. 
Note that here $B_{l} \simeq \mathcal{H}_{l}\left(q^{-2}\right)$ is in general not a subalgebra of $e_{l} B M W_{r} e_{l}$ - in contrast to the situation for Brauer algebras.

2.4. Partition algebras. The third family of examples are the partition algebras, introduced by Martin [22]. Like Brauer algebras, partition algebras are iterated inflations of group algebras of symmetric groups (see Xi [24]). For $r \in \mathbb{N}$ and $\delta \in k$, the partition algebra $P_{k}(r, \delta)$ has $k$-basis the set of all partitions of $2 r$ points. Such a partition may be drawn as a diagram with $2 r$ points arranged in two equal rows, and edges between points so that a pair of points is joined by a path of edges if and only if they lie in the same block of the partition. Note that different diagrams can represent the same partition. Multiplication is given by concatenation of diagrams, and any block of the concatenated diagram that does not contain a point from either the top or bottom row is replaced by $\delta$. This is independent of the diagrams chosen to represent the partitions. For more details see $[6,22]$. Xi defines $J_{l}$ to be the subspace of $P_{k}(r, \delta)$ spanned by all partitions whose diagrams have at most $l$ blocks containing a point from both top and bottom rows. In this way he obtains a chain of two-sided ideals:

$$
0 \subsetneq J_{0} \subseteq J_{1} \subseteq \cdots \subseteq J_{r-1} \subseteq J_{r}=P_{k}(r, \delta)
$$

which realizes $P_{k}(r, \delta)$ as an iterated inflation, in particular:

$$
J_{l} / J_{l-1} \simeq k \Sigma_{l} \otimes V_{l} \otimes V_{l}
$$

for some vector space $V_{l}$, see [24, Section 4] for details. If $\delta=0$ then $P_{k}(r, \delta)$ is not cellularly stratified since $J_{0}^{2}=0$, and so condition (E) cannot be satisfied. However if $\delta \neq 0$ we choose idempotents:

$$
e_{0}=\delta^{-1}
$$
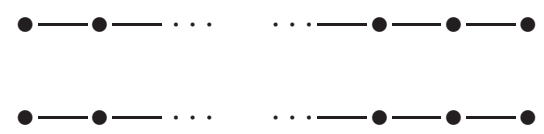

and for $l \in\{1,2, \ldots, r\}$ :

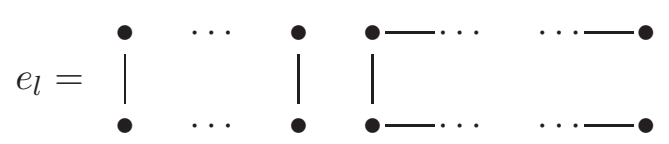

with $l$ vertical edges. We readily see that $P_{k}(r, \delta)$ is cellularly stratified. Note that the algebra $e_{l} P_{k}(r, \delta) e_{l}$ is isomorphic to $P_{k}(l, \delta)$ of which $k \Sigma_{l}$ is a subalgebra.

Proposition 2.6. Let $k$ be any field, $r$ an integer and $\delta \in k$. Suppose $\delta \neq 0$. Then the partition algebra $P_{k}(r, \delta)$ is cellularly stratified.

\section{Corner split quotients for Cellularly stratified algebras}

We assume the set-up as in Definition 2.1. In this section, we will set up functors $G_{l}: B_{l}-\bmod \rightarrow A-\bmod$, following the 'split pairs' approach developed in [4]. As background, we first recall some definitions. Let $\mathcal{C}$ and $\mathcal{D}$ be two module categories. Let $F, G$ be additive functors with $F: \mathcal{C} \rightarrow \mathcal{D}$ and $G: \mathcal{D} \rightarrow \mathcal{C}$.

Definition 3.1. The pair of functors $(F, G)$ is a split pair of functors if the composition $F \circ G$ is an autoequivalence of the category $\mathcal{D}$. If the two functors are exact then we say $(F, G)$ is an exact split pair of functors. 
It is shown in [4] that all exact split pairs are compositions of so-called corner split quotients with Morita equivalences. Next we recall the definition of a corner split quotient. Let $C$ and $D$ be rings. We call $D$ a split quotient of $C$ if $D$ is a subring of $C$, via an embedding $\varepsilon$ sending the unit of $D$ to that of $C$, and also there exists a surjective homomorphism $\pi: C \rightarrow D$, such that the composition $\pi \circ \varepsilon$ is the identity on $D$. The homomorphisms $\pi$ and $\varepsilon$ respectively induce two exact functors

$$
F={ }_{D} C \otimes_{C}-\quad \text { and } \quad G={ }_{C} D \otimes_{D}-
$$

between the categories $C$-mod and $D$-mod, namely restriction and inflation. The composition $F \circ G$ is the identity on $D$-mod, and hence $(F, G)$ is a split pair of functors.

Definition 3.2. Let $C$ be a ring, $e$ an idempotent, and $D$ a split quotient of $e C e$ viewed as a subring of $e C e$. Then we call $D$ a corner split quotient of $C$ with respect to $e$ if there is a left $C$-and right $e C e$-module $S$, which is projective as a right $D$-module via the embedding of $D$ into $e C e$, and which satisfies $e S \simeq D$ as left $D$-modules.

Note that every $D$-module is an eCe-module via the quotient map. Thus, in the definition, we may equivalently require $S$ just to be a right $D$-module. If $D$ is a corner split quotient of $C$ with respect to $e$, then the functors

$F=$ resoe $C \otimes_{C}-: C$-mod $\rightarrow e C e-\bmod \rightarrow D$-mod, $\quad G=S \otimes_{D^{-}}: D$-mod $\rightarrow C$-mod form an exact split pair (see [4, Lemma 3.2]).

Definition 3.3. Let $A$ be cellularly stratified. For each $l=1, \ldots, n$, define $S_{l}=$ $A e_{l} \otimes_{e_{l} A e_{l}} B_{l}$. We will refer to the functors $G_{l}:=S_{l} \otimes_{e_{l} A e_{l}}-: B_{l}$-mod $\rightarrow A$-mod as induction functors.

Note for each $B_{l}$-module $X$, we have $B_{l} \otimes_{B_{l}} X \simeq B_{l} \otimes_{e_{l} A e_{l}} X$, where $e_{l} A e_{l}$ acts on $X$ and $B_{l}$ by the quotient map $e_{l} A e_{l} \rightarrow B_{l}$.

Lemma 3.4. With the notation as above, $S_{l}$ is an $A$ - $B_{l}$-bimodule, and as such is isomorphic to $\left(A / J_{l-1}\right) e_{l}$, which gets its right $B_{l}$-module structure via the isomorphism in Lemma 2.3. In particular, the left A-module structure on $S_{l}$ factors through the quotient map $A \rightarrow A / J_{l-1}$.

Proof. Recall that by Lemma 2.3 and Lemma 2.2, $B_{l} \simeq e_{l}\left(A / J_{l-1}\right) e_{l}$ and $J_{l-1}=$ $A e_{l-1} A$ with $e_{l-1} e_{l}=e_{l-1}=e_{l} e_{l-1}$. Hence

$$
\begin{aligned}
J_{l-1} e_{l} \otimes_{e_{l} A e_{l}} B_{l} & =A e_{l}\left(e_{l-1} e_{l} A e_{l}\right) \otimes_{e_{l} A e_{l}} B_{l} \\
& =A e_{l} \otimes_{e_{l} A e_{l}} e_{l-1}\left(e_{l} A e_{l}\right)\left(e_{l}\left(A / J_{l-1}\right) e_{l}\right) \\
& =A e_{l} \otimes_{e_{l} A e_{l}} e_{l-1}\left(e_{l} A e_{l} / e_{l} J_{l-1} e_{l}\right)=0 .
\end{aligned}
$$

This implies the following isomorphism of $A$ - $B_{l}$-bimodules:

$S_{l} \simeq\left(A / J_{l-1}\right) e_{l} \otimes_{e_{l} A e_{l}} B_{l} \simeq\left(A / J_{l-1}\right) e_{l} \otimes_{e_{l}\left(A / J_{l-1}\right) e_{l}} B_{l} \simeq\left(A / J_{l-1}\right) e_{l} \otimes_{B_{l}} B_{l} \simeq\left(A / J_{l-1}\right) e_{l}$.

Proposition 3.5. Let $A$ be cellularly stratified. For each $l=1, \ldots, n$, the right $B_{l^{-}}$ module $S_{l}$ is free of rank $\operatorname{dim} V_{l}$. The algebra $B_{l}$ is a corner split quotient of $A / J_{l-1}$ with respect to $e_{l}$, realized by $S_{l}$. Hence there is an exact split pair situation relating $A / J_{l-1}$ and $B_{l}$ via the $A / J_{l-1}-B_{l}$-bimodule $S_{l}$. 
Proof. Assume $l=1$, that is we consider the lowest layer of $A$. For convenience we will omit the subindices 1 , that is, we use $B=B_{1}, V=V_{1}, u=u_{1}, v=v_{1}$ and $e=e_{1}$. Then we have an isomorphism $B \rightarrow e A e=B \otimes u \otimes v$, mapping $b \in B$ to $b \otimes u \otimes v$, in particular mapping the unit of $B$ to $e$. So $B$ is a split quotient of $e A e$. Let $S$ denote the $A-e A e$-bimodule $A e$. Certainly as left $B$-modules, we have $e S \simeq B$. Thus to show that $B$ is a corner split quotient of $A$, it remains to prove that $S$ is a projective right $B$-module. We will do so by showing that $S$ is free as a right $B$-module.

As a right $B$-module, $S=A e$ is isomorphic to $B \otimes V \otimes v$, with the right action of $b \in B$ given by multiplication with $b \otimes u \otimes v$. Take a basis $\left\{x_{i}\right\}$ of $V$. Then for each basis element $x_{i}$ we have

$$
\left(c \otimes x_{i} \otimes v\right) \cdot(b \otimes u \otimes v)=\left(c \cdot b \otimes x_{i} \otimes v\right) .
$$

This says that $B \otimes x_{i} \otimes v$ is isomorphic as a right $B$-module to the regular representation $B_{B}$, and so $S=B \otimes V \otimes v$ is a direct sum of $\operatorname{dim}(V)$ many copies of $B_{B}$. This shows the claim for $l=1$.

Now, for $l=1, \ldots, n, A / J_{l-1}$ is cellularly stratified by Lemma 2.2. Hence we obtain from the above that $B_{l}$ is a corner split quotient of $A / J_{l-1}$ with respect to $e_{l}$ realized by the $A / J_{l-1}-B_{l}$-bimodule $S_{l}=\left(A / J_{l-1}\right) e_{l}$.

Remark. (a) We remarked in Section 2.1 that for a cellularly stratified algebra $A$ the quotient $A / J_{l}$ is again cellularly stratified. The induction functors associated to one fixed layer of $A$ and to the corresponding layer of $A / J_{l}$ are the same. More precisely, for $l=1$ and the idempotent $e_{2} \in J_{2}$ we have the associated induction functor $G_{2}$ : $B_{2}$-mod $\rightarrow A$-mod. Similarly, for the cellularly stratified quotient $A / J_{1}$, we have without shifting the labels - a functor $\bar{G}_{2}: B_{2}$-mod $\rightarrow A / J_{1}$-mod. The functor $G_{2}$ is given by tensoring with $A e_{2} \otimes_{e_{2} A e_{2}} B_{2}$. By the proof of Lemma 3.4, this is the same as tensoring with $\left(A e_{2} / J_{1} e_{2}\right) \otimes_{e_{2} A e_{2} / e_{2} J_{1} e_{2}} B_{2}$. Hence the following diagram commutes, and we will no longer distinguish $G_{2}$ and $\bar{G}_{2}$ in our notation:

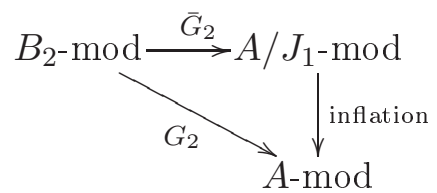

(b) If $A$ is cellularly stratified and, in addition, $B_{l}$ is a subalgebra of $e_{l} A e_{l}$ then the algebra $B_{l}$ is a corner split quotient of $A$ with respect to $e_{l}$, realized by the bimodule $A e_{l} \otimes_{e_{l} A e_{l}} B_{l}$. This may be seen by slightly adapting the proof of Proposition 3.5. Hence, in this case, there is an exact split pair situation relating $A$ and $B_{l}$. This is the case in the examples of the Brauer algebra and the partition algebra. In particular, this gives a functor $F_{l}=e_{l} A \otimes_{A}-: A$-mod $\rightarrow B_{l}$-mod. However, in general, $B_{l}$ is not necessarily a corner split quotient of $A$, since our axioms do not require $B_{l}$ to be a subalgebra of $e_{l} A e_{l}$. Indeed, this does not hold in the example of the BMW algebra. 


\section{Properties of the induction functor $G$}

Next we collect some first properties of the induction functor $G_{l}$. We will see throughout this paper that $G_{l}$ transfers the structure of the small cellular algebras $B_{l}$ to the inflated algebra $A$. In this section we will see in particular that the functor $G_{l}$ sends cell modules to cell modules, and each cell module of the cellularly stratified algebra $A$ is obtained in this way. In the case of $A$ being a Brauer algebra, the functor $G_{l}$ defined here does precisely the same as the combinatorial induction process used in [12, Section 5] to produce cell modules for Brauer algebras from Specht modules of symmetric groups. Continuing the notation of the previous section, we omit subindices.

Proposition 4.1. The induction functor $G$ has the following properties:

(1) The functor $G$ is exact.

(2) Let $X$ be any $B$-module. Then $G(X)=X \otimes_{k} V$ as a vector space.

Proof. Exactness of the functor $G$ is implied by $S$ being projective, see Proposition 3.5. Next, let $X$ be a left $B$-module. Using Lemma $2.3, X$ is an $e A e$-module by inflation and moreover $e J^{\prime} e \cdot X=0$. Then:

$$
\begin{aligned}
G(X) & =A e \otimes_{e A e} B \otimes_{B} X \\
& =A e \otimes_{e A e} X \\
& =\left(A e / J^{\prime} e\right) \otimes_{e A e} X \quad \text { by Equation (5) } \\
& =\left(A e / J^{\prime} e\right) \otimes_{B} X \\
& \simeq\left(B^{\operatorname{dim} V}\right) \otimes_{B} X \quad \text { by Proposition 3.5, } \\
& \simeq X^{\operatorname{dim} V} \simeq X \otimes_{k} V
\end{aligned}
$$

as vector spaces.

Let us fix some notation here that will be valid throughout the article. Any cellular algebra $A$ comes equipped with a set of cell modules $\Theta_{A}(\lambda)$ with $\lambda$ in some index set $\Lambda_{A}$. Then a complete set of simple $A$-modules is given by the modules $L_{A}(\lambda)$ for

$\lambda \in \Lambda_{A}^{\text {simple }} \subseteq \Lambda_{A}$. If there is no doubt about the algebra concerned, we will just write $\Theta(\lambda)$ or $L(\lambda)$.

The next result assumes $A$ to be cellularly stratified and then explains how the induction functor $G$ relates the given cellular structures of $A$ and $B_{l}$ (for each $l$ ).

Proposition 4.2. Suppose $A$ is cellularly stratified. Then the functor $G$ sends the cell modules of $B$ to cell modules of $A$, and each cell module of $A$ is obtained in this way. In particular, taking disjoint sets $\Lambda_{B_{l}}$ to label the sets of cell modules for the cellular algebras $B_{l}$, the labels of the cell modules of $A$ are the elements of $\Lambda_{A}=\bigcup_{l=1}^{n} \Lambda_{B_{l}}$.

Proof. (a) The cell filtration of the layer $J / J^{\prime}$ is produced from that of $B$, see the description of the inflation technique in [18]. More precisely: By analogy with the functor $G=S \otimes_{B}-: B$-mod $\rightarrow A$-mod, we define the functor $G^{\prime}=-\otimes_{B} S^{\prime}$ : $\bmod -B \rightarrow \bmod -A$ with $S^{\prime}=B \otimes_{e A e} e A$. Then $G$ and $G^{\prime}$ are exact by Proposition 4.1. 
Let $\{0\}=I_{0} \subseteq I_{1} \subseteq \ldots \subseteq I_{s}=B$ be a cell chain of $B$. This means there exist left $B / I_{t-1}$-modules $\Theta_{B}(t)$ such that the subquotients are given by the $B / I_{t-1}$-bimodule

$$
I_{t} / I_{t-1} \simeq \Theta_{B}(t) \otimes i\left(\Theta_{B}(t)\right)
$$

where $i$ is the involution of the cellular algebra $B$. We tensor from the left with $S$ and from the right with $S^{\prime}$ and get the chain of $A$ - $A$-bimodules

$$
S \otimes_{B} I_{0} \otimes_{B} S^{\prime} \subseteq S \otimes_{B} I_{1} \otimes_{B} S^{\prime} \subseteq \ldots \subseteq S \otimes_{B} I_{s} \otimes_{B} S^{\prime}=S \otimes_{B} B \otimes_{B} S^{\prime} \simeq J / J^{\prime}
$$

where the last isomorphism is explained in (i) below. The subquotients of this ideal chain are given by the $A / J^{\prime}$-bimodules

$$
S \otimes_{B}\left(I_{t} / I_{t-1}\right) \otimes_{B} S^{\prime} \simeq\left(S \otimes_{B} \Theta_{B}(t)\right) \otimes_{k} i\left(S \otimes_{B} \Theta_{B}(t)\right)
$$

which we show in (ii) below. This provides filtrations for all layers $B_{l} \otimes V_{l} \otimes V_{l}$ with $1 \leq l \leq n$, and hence this provides a cell chain of $A$. This implies that the cell modules of $A$ are given by $G\left(\Theta_{B}(t)\right)$.

(i) By Equation (3), we have $\left(J / J^{\prime}\right) e=B \otimes V \otimes v$, and similarly, $e\left(J / J^{\prime}\right)=B \otimes u \otimes V$. By Equation (5), $S \otimes_{B} B \otimes_{B} S^{\prime} \simeq\left(J / J^{\prime}\right) e \otimes_{B} e\left(J / J^{\prime}\right)$. Define

$$
\varphi:\left(J / J^{\prime}\right) e \otimes_{B} e\left(J / J^{\prime}\right) \rightarrow J / J^{\prime}
$$

to be the map given by multiplication. This map is surjective since $J=J e J$. Moreover, $\left(J / J^{\prime}\right) e$ and $e\left(J / J^{\prime}\right)$, as right and left $B$-modules respectively, are free of $\operatorname{rank} \operatorname{dim} V$, see the proof of Proposition 3.5. Hence

$$
\operatorname{dim}\left(\left(J / J^{\prime}\right) e \otimes_{B} e\left(J / J^{\prime}\right)\right)=\operatorname{dim}\left(B^{(\operatorname{dim} V)^{2}}\right)=\operatorname{dim} B \otimes V \otimes V=\operatorname{dim} J / J^{\prime},
$$

and so $\varphi$ is an isomorphism. This shows that $S \otimes_{B} B \otimes_{B} S^{\prime} \simeq J / J^{\prime}=B \otimes V \otimes V$.

(ii) We show that the subquotients have the form claimed in Equation (7). Recall that the involution $i$ of the algebra $A$ operates as follows in the layer $B \otimes V \otimes V$ : $i(b \otimes x \otimes y)=i(b) \otimes y \otimes x$ where $i(b)$ denotes the operation of the involution of $B$ on element $b \in B$. Let $d \otimes x \otimes v \in \Theta_{B}(t) \otimes V \otimes v$. Then $i(d \otimes x \otimes v)=i(d) \otimes v \otimes x$, and hence

$$
i\left(\Theta_{B}(t) \otimes V \otimes v\right)=i\left(\Theta_{B}(t)\right) \otimes v \otimes V \simeq i\left(\Theta_{B}(t)\right) \otimes u \otimes V,
$$

as right modules over $A / J^{\prime}$.

We finish this section by comparing homomorphism spaces.

Proposition 4.3. For all $B$-modules $X$ and $Y, \operatorname{Hom}_{B}(X, Y)=\operatorname{Hom}_{A}(G X, G Y)$ where $G$ is the functor $S \otimes_{B}-$ for $S=A e \otimes_{e A e} B$. In particular, $G X$ is indecomposable if and only if $X$ is so.

Proof. Indeed, using the adjointness of the Hom functor and the tensor functor, we have

$$
\begin{aligned}
\operatorname{Hom}_{A}(G X, G Y) & =\operatorname{Hom}_{A}\left(A e \otimes_{e A e} B \otimes_{B} X, A e \otimes_{e A e} B \otimes_{B} Y\right) \\
& =\operatorname{Hom}_{A}\left(A e \otimes_{e A e} X, A e \otimes_{e A e} Y\right) \\
& \simeq \operatorname{Hom}_{e A e}\left(X, \operatorname{Hom}_{A}\left(A e, A e \otimes_{e A e} Y\right)\right) \\
& =\operatorname{Hom}_{e A e}\left(X, e A e \otimes_{e A e} Y\right) \\
& =\operatorname{Hom}_{e A e}(X, Y)=\operatorname{Hom}_{B}(X, Y) .
\end{aligned}
$$


Here the last equality holds as $X$ and $Y$ are $B$-modules, and $e A e$-modules via inflation.

Remark. Split pairs do not in general produce equalities of Ext ${ }^{1}$-groups: For instance, assume $A$ is not semisimple, and $B$ is its maximal semisimple quotient which occurs in $A$ as subalgebra. Then $A$ and $B$ form a split pair. The induction functor $G$ in this situation is just inflation. Choose simple $B$-modules $S, T$ such that there exist a nonsplit extension between $G(S)$ and $G(T)$. Then $0=\operatorname{Ext}_{B}^{1}(S, T) \neq \operatorname{Ext}_{A}^{1}(G(S), G(T))$. Thus in order to compare Ext ${ }^{1}$-groups, we need to use some stronger properties, for example the special choice of $S$ and of the idempotent $e$. Indeed, in this case we then can identify extension groups in all degrees, see Section 7.

\section{A structural Characterization of Cellularly stratified Algebras}

Diagram algebras are defined in a combinatorial way. Their cellular structure has typically been identified by writing them as iterated inflations of known cellular algebras. The definition of a cellularly stratified algebra naturally enhances the definition of an iterated inflation. In this section we discuss how the combinatorial setup of Definition 2.1 is reflected by structural properties. We define a set of properties, $(\mathrm{G}),(\mathrm{J})$ and $(\mathrm{F})$. The structure theory of cellularly stratified algebras described in this article is really based on this new set of properties. We will see in the first section, that under the (mild) assumption of the involution $i$ fixing the given idempotents, the new set of properties characterizes cellularly stratified algebras (see Proposition 5.1). This covers all generic cases (that is $\delta \neq 0$ ) among the examples given in Section 2. This characterization is then improved to full generality in the following two sections (see Proposition 5.2), including the non-generic cases, modifying properties $(\mathrm{J})$ and $(\mathrm{F})$.

5.1. Generic case. We define the following structural properties:

(G) Let $A$ be a finite dimensional algebra over a field $k$ with an involutory antiisomorphism $i: A \rightarrow A$. Suppose there is a set of idempotents $\left\{e_{l}: l=1, \ldots, n\right\}$ for some natural number $n$, such that $e_{n}=1$ and $e_{l} e_{m}=e_{m}=e_{m} e_{l}$ if $l>m$.

(J) For each $l=1, \ldots, n$, let $J_{l}:=A e_{l} A$ and $B_{l}:=e_{l} A e_{l} / e_{l} J_{l-1} e_{l}$. Suppose $e_{l}=$ $i\left(e_{l}\right)$, and the algebra $B_{l}$ is cellular with respect to the involution $i$. Moreover, suppose that, as a vector space, $J_{l}$ has a decomposition $J_{l}=J_{l-1} \oplus X_{l}$ for some subspace $X_{l}=i\left(X_{l}\right)$ for $l=2, \ldots, n$.

(F) For each $l=1, \ldots, n$, define the $A$ - $B_{l}$-bimodule $S_{l}:=A e_{l} / J_{l-1} e_{l}$. Assume that for each $l=1, \ldots, n$, module $S_{l}$ is free of finite rank over $B_{l}$ and multiplication induces an isomorphism of $A$ - $A$-bimodules

$$
A e_{l} / J_{l-1} e_{l} \otimes_{B_{l}} e_{l} A / e_{l} J_{l-1} \simeq A e_{l} A / A e_{l-1} A=J_{l} / J_{l-1} .
$$

Proposition 5.1. (a) Assume that $A$ is a cellularly stratified algebra with $i\left(e_{l}\right)=e_{l}$ for $l=1, \ldots, n$. Then $A$ satisfies properties $(G),(J)$ and $(F)$.

(b) Suppose algebra A satisfies properties $(G),(J)$ and $(F)$. Then $A$ is cellularly stratified with respect to the given algebras $B_{l}$ and idempotents $e_{l}$. 
Proof. (a) Let $A$ be cellularly stratified and assume moreover that the involution $i$ fixes all idempotents $e_{l}$. The general assumption $(\mathrm{G})$ is satisfied by definition. Assume that $I$ is an ideal in the cell chain of $A$. Then, by the definition of cellularity, there exists a vector space $Y$ such that $A=I \oplus Y$ with $i(Y)=Y$. By Lemma 2.2, all $J_{l}$ occur as ideals in a cell chain of $A$. By Lemma 2.3, it follows that assumption $(\mathrm{J})$ is satisfied. By Proposition 3.5, $S_{l}$ is a free right $B_{l}$-module of finite rank, and the isomorphism in property $(\mathrm{F})$ has been shown in the proof of Proposition 4.2, part (i).

(b) Assume the structural properties (G), (J) and (F) are satisfied. We now construct a cell chain of $A$ as in the proof of Proposition 4.2. By property (F), $S_{l}=e_{l} A / e_{l} J_{l-1}$ is free as right $B_{l}$-module, and since $i\left(e_{l}\right)=e_{l}$, this implies $i\left(S_{l}\right)=e_{l} A / e_{l} J_{l-1}$ is free as left $B_{l}$-module. Fix a cell chain $\{0\}=I_{0} \subseteq I_{1} \subseteq \ldots \subseteq I_{s}=B_{l}=B$. Denote by $\Theta_{B}(t)$ the cell modules of $B$. Then we obtain for all $t$ the isomorphism

$$
S_{l} \otimes_{B}\left(I_{t} / I_{t-1}\right) \otimes_{B} i\left(S_{l}\right) \simeq\left(S_{l} \otimes_{B} \Theta_{B}(t)\right) \otimes_{k} i\left(S_{l} \otimes_{B} \Theta_{B}(t)\right) .
$$

By $(\mathrm{F})$, multiplication provides an isomorphism

$$
S_{l} \otimes_{B} B \otimes_{B} i\left(S_{l}\right)=A e_{l} / J_{l-1} e_{l} \otimes_{B} B \otimes_{B} e_{l} A / e_{l} J_{l-1} \simeq A e_{l} A / A e_{l-1} A=J_{l} / J_{l-1} .
$$

Hence this constructs a cell chain of $A$. We next see that $A$ is an iterated inflation of the algebras $B_{l}$ : By $(\mathrm{F})$, we can write

$$
S_{l}=\bigoplus_{j=1}^{m} v_{j} B_{l}=V_{l} \otimes_{k} B_{l}
$$

as right $B_{l}$-module where $V_{l}$ is an $m$-dimensional vector space. Applying $i$ gives a similar decomposition,

$$
i\left(S_{l}\right)=\bigoplus_{j=1}^{m} B_{l} i\left(v_{j}\right)=B_{l} \otimes_{k} V_{l} .
$$

Hence $S_{l} \otimes_{B} B \otimes_{B} i(S)=V_{l} \otimes_{k} B \otimes_{k} V_{l}$ and so $A$ is an iterated inflation of cellular algebras $B_{l}$ along $V_{l}$. The condition $e_{l}=1_{B_{l}} \otimes u_{l} \otimes v_{l}$ is implied by (F) as follows. We can choose $v_{1}=e_{l}$, then under the isomorphism

$$
J_{l} / J_{l-1} \simeq S_{l} \otimes_{B_{l}} B_{l} \otimes_{B_{l}} i\left(S_{l}\right)
$$

the element $e_{l}$ is mapped to $e_{l} \otimes 1_{B_{l}} \otimes e_{l}=v_{1} \otimes 1_{B_{l}} \otimes v_{1}$ as required. So $A$ is cellularly stratified.

Hence the above structural assumptions may be taken as an alternative, slightly less general, starting point of the theory of cellularly stratified algebras, avoiding the explicit use of iterated inflations. The examples given in Section 2 show that the assumption $i\left(e_{l}\right)=e_{l}$ is not satisfied in some exceptional cases, namely where the parameter $\delta=0$. We discuss these cases in the following subsections.

5.2. Modifying property (J). In the examples in Section 2, assumption $(\mathrm{J})$ is almost always satisfied. In the exceptional cases when the parameter $\delta=0$, the idempotents $e_{l}$ are not fixed under the involution $i$. These cases fit into the following setup:

Let $\Lambda$ be an algebra with idempotent $e$ and involutory anti-automorphism $i$ such that $e$ and $i(e)$ are equivalent. The equivalence of idempotents implies that there are elements 
$p, q \in \Lambda$ such that $e=p q$ and $i(e)=q p$. Applying $i$ also gives $i(e)=i(q) i(p)$ and $e=i(p) i(q)$. Assume that $p$ is fixed under $i$. Then there is an algebra isomorphism $\varphi: i(e) i(\Lambda) i(e) \rightarrow e i(\Lambda) e=e \Lambda e$, sending $x$ to $p x q$, that is $i(e) i(a) i(e)=q p i(a) q p$ to $p q p i(a) q p q=e p i(a) q e$. The inverse of $\varphi$ multiplies by $q$ from the left and by $p$ from the right. Now define $j:=\varphi \circ\left(\left.i\right|_{e \Lambda e}\right): e \Lambda e \rightarrow e \Lambda e$. Then $j$ is an anti-automorphism with

$$
j^{2}(x)=\varphi(i(p i(x) q))=\varphi(i(q) x i(p))=p i(q) x i(p) q=i(p) i(q) x p q=e x e=x
$$

for any $x \in e \Lambda e$. Hence $j$ is an involution on $e \Lambda e$. We now iterate this construction. Assume that $A$ is a finite dimensional algebra over $k$ satisfying property $(\mathrm{G})$ such that the idempotents $e_{l}$ and $i\left(e_{l}\right)$ are equivalent for all $l$. Let $J_{l-1}=A e_{l-1} A$, define $\Lambda=A / J_{l-1}$ and define $e$ to be the image of $e_{l}$ in $\Lambda$. The equivalence of the idempotents $e_{l-1}$ and $i\left(e_{l-1}\right)$ in $A$ implies that the ideal $J_{l-1}$ is fixed under $i$. So $i$ is defined on $\Lambda$. Then $e$ and $i(e)$ are equivalent in $\Lambda$. This in turn implies that there are elements $p, q \in \Lambda$ such that $e=p q$ and $i(e)=q p$. Now we assume that $p$ is fixed under $i$, and $B_{l}=e \Lambda e$ is cellular with respect to $j_{l}=j$. We modify property $(\mathrm{J})$ accordingly:

$\left(\mathrm{J}^{\prime}\right)$ For each $l=1, \ldots, n$, let $J_{l}:=A e_{l} A$ and $B_{l}:=e_{l} A e_{l} / e_{l} J_{l-1} e_{l}$. Suppose, for each $l=1, \ldots, n$, that there exist elements $p_{l}, q_{l} \in A$ such that $e_{l}=p_{l} q_{l}$, $i\left(e_{l}\right)=q_{l} p_{l}$ modulo $J_{l-1}$, and that $i\left(p_{l}\right)=p_{l}$, and that $B_{l}$ is cellular with respect to $j_{l}=\varphi \circ\left(i_{\mid B_{l}}\right)$. Moreover, suppose that, as a vector space, $J_{l}$ has a decomposition $J_{l}=J_{l-1} \oplus X_{l}$ for some subspace $X_{l}$ which is fixed by the involution $i$, for $l=2, \ldots, n$.

Note $(\mathrm{J})$ implies $\left(\mathrm{J}^{\prime}\right)$ by choosing $p=q=e$, but the two properties are not equivalent.

Remark. Suppose we are given elements $p, q \in A$ as above with $i(p)=p$ and $e=p q$ and $i(e)=q p$ modulo lower layers. Calculating modulo lower layers,

$$
i(q p q)=i(p q) i(q)=q p i(q)=q i(p) i(q)=q i(q p)=q p q .
$$

Assume in addition that $q p q=c e$ for some scalar $c \in k^{\times}$. Then $c e=q p q=i(q p q)=$ $i(c e)=c i(e)$ modulo lower layers, and hence $e=i(e)$ modulo lower layers.

Examples. We return to the examples in Section 2 and illustrate the modified property $\left(\mathrm{J}^{\prime}\right)$ in the exceptional cases. Consider the Brauer algebra with parameter $\delta=0$ when $r$ is odd. Then

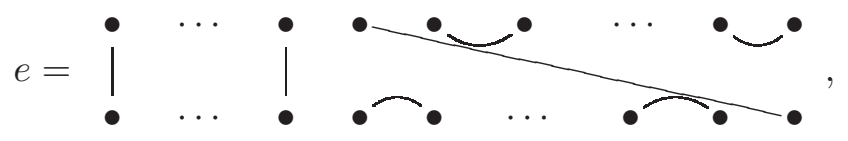

and we can choose

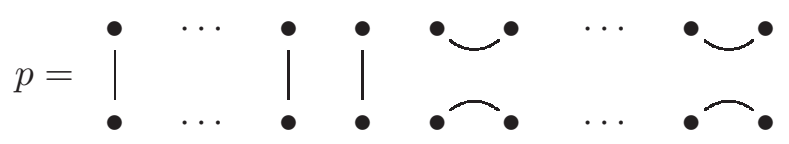

and

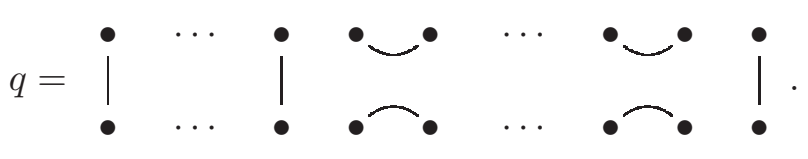


These choices are not unique, alternatively one can take $p$ as above and

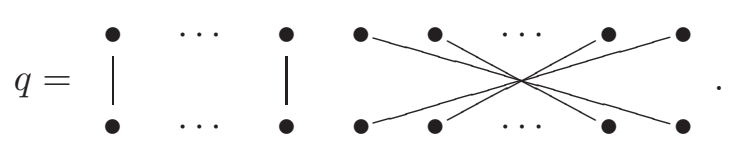

The corresponding case of the BMW algebras is handled in precisely the same way. So all exceptional cases from the examples in Section 2 are covered by the modified property $\left(\mathrm{J}^{\prime}\right)$.

Remark. In general, if $A$ is cellularly stratified, then by property (E), $e_{l}=1_{B_{l}} \otimes u_{l} \otimes v_{l}$ is an idempotent and hence by the remark after Definition 2.1, $\varphi\left(v_{l}, u_{l}\right)=1=\varphi\left(u_{l}, v_{l}\right)$. Define $p=1 \otimes u_{l} \otimes u_{l}$ and $q=1 \otimes v_{l} \otimes v_{l}$. Then by the multiplication, given in Equation (3), $p q=e+$ lower terms and $q p=i(e)+$ lower terms. Moreover, by Equation $(2), i(p)=p$ and $i(q)=q$. So, modulo lower layers, we have $e=p q, i(e)=q p$ with $p=i(p)$ and $q=i(q)$. Hence cellularly stratified algebras satisfy property $\left(\mathrm{J}^{\prime}\right)$ as well.

5.3. Modifying property (F). Assuming properties $(\mathrm{G}),(\mathrm{F})$ and $\left(\mathrm{J}^{\prime}\right)$ for an algebra $A$ will not be sufficient for $A$ to be cellularly stratified with respect to the chosen idempotents $e_{l}$, as we will see in an example at the end of this chapter. The only obstacle is to show that the idempotents are of the form $e_{l}=1 \otimes u_{l} \otimes v_{l}$ for some $u_{l}, v_{l} \in V_{l}$. We will now describe how to strengthen property (F) to really obtain an equivalence in this general situation:

$\left(\mathrm{F}^{\prime}\right)$ For each $l=1, \ldots, n$, define the $A$ - $B_{l}$-bimodule $S_{l}:=A e_{l} / J_{l-1} e_{l}$. Assume that $S_{l}$ is free of finite rank over $B_{l}$ for each $l=1, \ldots, n$, and there exists a direct sum decomposition of $S_{l}$ in which $e_{l}$ and $q_{l} e_{l}$ generate free summands such that $q_{l} e_{l}=c e_{l}$ for some $c \in k^{\times}$if the summands coincide. Assume moreover multiplication induces an isomorphism of $A$ - $A$-bimodules

$$
A e_{l} / J_{l-1} e_{l} \otimes_{B_{l}} e_{l} A / e_{l} J_{l-1} \simeq A e_{l} A / A e_{l-1} A=J_{l} / J_{l-1} .
$$

Note that $\left(\mathrm{F}^{\prime}\right)$ implies $(\mathrm{F})$, and in case $e_{l}=i\left(e_{l}\right)$, we can choose $e_{l}=p_{l}=q_{l}=$ $1_{B} \otimes u_{l} \otimes u_{l}$ for some $u_{l} \in V_{l}$, so $\left(\mathrm{F}^{\prime}\right)$ is just the same as $(\mathrm{F})$. Now we can state the structural characterization of cellularly stratified algebras in the general case:

Proposition 5.2. An algebra $A$ is cellularly stratified if and only if it satisfies properties $(G),\left(J^{\prime}\right)$ and $\left(F^{\prime}\right)$ as stated above.

Proof. (a) Let $A$ be cellularly stratified. Then (G) holds. Assume $e_{l}=1_{B_{l}} \otimes u_{l} \otimes v_{l}$, and define $q_{l}=1_{B_{l}} \otimes v_{l} \otimes v_{l}$ and $p_{l}=1_{B_{l}} \otimes u_{l} \otimes u_{l}$. Then by the remark at the end of Section 5.2, property $\left(\mathrm{J}^{\prime}\right)$ holds. Moreover, calculating modulo lower layers,

$$
q_{l}\left(e_{l} A e_{l} / e_{l} J_{l-1} e_{l}\right)=q_{l} \cdot\left(B_{l} \otimes u_{l} \otimes v_{l}\right)=B_{l} \otimes v_{l} \otimes v_{l},
$$

which is a free direct summand of $B_{l} \otimes V_{l} \otimes v_{l}$. We distinguish two cases. If $v_{l}=\lambda u_{l}$ for some scalar $\lambda$, then $B_{l} \otimes u_{l} \otimes v_{l}=B_{l} \otimes v_{l} \otimes v_{l}$ with $q_{l}=\lambda e_{l}$, and hence $q_{l} e_{l}=\lambda e_{l}$ for some scalar $\lambda$. If the vectors $\left\{u_{l}, v_{l}\right\}$ are linearly independent, then $B_{l} \otimes u_{l} \otimes v_{l} \cap B_{l} \otimes v_{l} \otimes v_{l}=0$. Hence property $\left(\mathrm{F}^{\prime}\right)$ holds. 
(b) We assume that the algebra $A$ satisfies properties $(\mathrm{G}),\left(\mathrm{J}^{\prime}\right)$ and $\left(\mathrm{F}^{\prime}\right)$. For simplicity, we will restrict ourselves here to the lowest layer and suppress indices. Thus $J=A e A$, $B=e A e$ and the idempotent $e$ is of the form $e=p q$ with $i(e)=q p$, where $i(p)=p$. For preparation, note that $e A=p i(e) A$ and $e p A q e=e A e$. For example since $p A \subseteq A$, we obtain $p q p A \subseteq p q A=e A$. Similarly, $q A \subseteq A$ implies that $e A=p q p q A \subseteq p q p A$. Hence we can write $e A=p i(e) A$. By a similar argument we obtain $e p A q e=e A e$.

By property $\left(\mathrm{F}^{\prime}\right)$ we can write

$$
S=A e=\bigoplus_{j=1}^{m} v_{j}(e A e)
$$

as right $e A e$-modules for some $v_{1}, \ldots, v_{m} \in A e$. Applying $i$, we get a corresponding decomposition

$$
i(e) A=i(A e)=\bigoplus_{j=1}^{m} i(e) A i(e) i\left(v_{j}\right)
$$

as left $i(e) A i(e)$-modules. Note that $p i(e) A i(e)=e p A q e p=e A e p$. Since $e A=p i(e) A$, we obtain the following decomposition of $e A$ as a left $e A e$-module:

$$
e A=\bigoplus_{j=1}^{m} p i(e) A i(e) i\left(v_{j}\right)=\bigoplus_{j=1}^{m} e A e \cdot p i\left(v_{j}\right),
$$

and so $e A$ is a free left $e A e$-module with basis $p i\left(v_{j}\right)$ for $j=1, \ldots, m$. It follows from $\left(\mathrm{F}^{\prime}\right)$, that we have an isomorphism

$$
\begin{aligned}
J & =A e A \simeq A e \otimes_{e A e} e A e \otimes_{e A e} e A \\
& \simeq \bigoplus_{j=1}^{m} v_{j} \cdot e A e \otimes_{e A e} e A e \otimes_{e A e} \bigoplus_{j=1}^{m} e A e \cdot p i\left(v_{j}\right) \\
& \simeq V \otimes_{B} B \otimes_{B} V
\end{aligned}
$$

where $V$ is a vector space of dimension $m$. Hence $A$ is an iterated inflation of cellular algebras $B_{l}$. The above isomorphism sends aeb to $a e \otimes e \otimes e b$. In particular, $e \in J$ is mapped to $e \otimes e \otimes e=e \otimes 1_{B} \otimes e$. By $\left(\mathrm{F}^{\prime}\right)$, we can choose the basis elements $v_{j}$ such that $v_{1}=e$. Recall the remark after the definition of property $\left(\mathrm{J}^{\prime}\right)$. If $q e=c e$ for some scalar $c \in k^{\times}$, then $e=i(e)$, and hence $e \in J$ is mapped to $v_{1} \otimes 1_{B} \otimes v_{1}$. If $e$ and qe generate different free direct summands of $A e$, then choose $v_{2}=q e=q p q=i(q p q)=i\left(v_{2}\right)$. Then $e \in J$ is mapped to $v_{1} \otimes 1_{B} \otimes p i\left(v_{2}\right)$. Hence $e$ is always of the required form, and $A$ is indeed cellularly stratified.

5.4. Examples. We end this chapter by discussing the above properties on two examples. In the first example, we consider an algebra with a set of idempotents satisfying properties $(\mathrm{G}),\left(\mathrm{J}^{\prime}\right)$ and $(\mathrm{F})$ where property $(\mathrm{J})$ and $\left(\mathrm{F}^{\prime}\right)$ do not hold. This algebra is not cellularly stratified with respect to the chosen stratification data. This shows that property $\left(\mathrm{F}^{\prime}\right)$ is necessary. However the algebra considered is cellularly stratified with respect to a differently chosen stratification data. In the second example we give an algebra that is cellularly stratified with respect to a particular iterated inflation, but not cellularly stratified with respect to isomorphic inflation data. 
(1) We consider the two-dimensional algebra $B$ with basis $a, b$, where $a^{2}=a, b^{2}=b$ and $a b=b a=0$, which is cellular with respect to the identity involution $i$. We take $V$ to be a three-dimensional vector space, with basis $v_{1}, v_{2}, v_{3}$, and set $J$ to be the inflation $B \otimes V \otimes V$ with multiplication given by:

$$
\left(c_{1} \otimes v_{i_{1}} \otimes v_{j_{1}}\right)\left(c_{2} \otimes v_{i_{2}} \otimes v_{j_{2}}\right)=\left(c_{1} \varphi\left(v_{j_{1}}, v_{i_{2}}\right) c_{2} \otimes v_{i_{1}} \otimes v_{j_{2}}\right),
$$

where $\varphi\left(v_{3}, v_{3}\right)=\varphi\left(v_{1}, v_{2}\right)=\varphi\left(v_{2}, v_{1}\right)=1$ and $\varphi\left(v_{i}, v_{j}\right)=0$ otherwise. This is compatible with the usual involution $i$ of an inflation. The algebra $A$ which we consider is obtained by adjoining a unit element to $J, A=J \oplus k 1_{A}$. We define

$$
e=\left(a \otimes v_{3} \otimes v_{3}\right)+\left(b \otimes v_{1} \otimes v_{2}\right),
$$

which is an idempotent. Then $i(e)=\left(a \otimes v_{3} \otimes v_{3}\right)+\left(b \otimes v_{2} \otimes v_{1}\right) \neq e$. We set $e_{2}=1_{A}$ and $e_{1}=e$. Property $(\mathrm{G})$ is seen to hold. Now we turn to property $\left(\mathrm{J}^{\prime}\right)$. We see that $A e_{1} A=A e A=J$ and $e A e=\operatorname{span}_{k}\left\{a \otimes v_{3} \otimes v_{3}, b \otimes v_{1} \otimes v_{2}\right\} \simeq B$. We let

$$
p=\left(1_{B} \otimes v_{3} \otimes v_{3}\right)+\left(1_{B} \otimes v_{1} \otimes v_{1}\right) \text { and } q=\left(a \otimes v_{3} \otimes v_{3}\right)+\left(b \otimes v_{2} \otimes v_{2}\right) .
$$

Then $e=p q$ and $i(e)=q p$ and $i(p)=p$. The induced involution we obtain on $e A e \simeq B$ is again the identity. The top layer presents no problems and property $\left(\mathrm{J}^{\prime}\right)$ is satisfied. For property $(\mathrm{F})$, we consider the $A$-eAe-bimodule $S_{1}=A e$. As a right $e A e$-module:

$$
\begin{aligned}
A e= & \operatorname{span}_{k}\left\{a \otimes v_{i} \otimes v_{3}, b \otimes v_{i} \otimes v_{2}: i=1,2,3\right\}, \\
= & \left\langle a \otimes v_{3} \otimes v_{3}, b \otimes v_{1} \otimes v_{2}\right\rangle \oplus\left\langle b \otimes v_{3} \otimes v_{2}, a \otimes v_{1} \otimes v_{3}\right\rangle \\
& \oplus\left\langle a \otimes v_{2} \otimes v_{3}, b \otimes v_{2} \otimes v_{2}\right\rangle
\end{aligned}
$$

The first summand is $e A e \simeq B$ and the second and third are each isomorphic to $e A e$ as right $e A e$-modules, thus $S_{1}$ is free of rank three as a right $e A e$-module. The top layer again poses no problems and property $(\mathrm{F})$ holds. Property $\left(\mathrm{F}^{\prime}\right)$ is, however, not satisfied and the idempotent $e_{1}=e$ is not of the form specified in property (E) of Definition 2.1.

The algebra $A$ is in fact cellularly stratified though: one must simply make a different choice of idempotents. Starting with the idempotents $1_{B} \otimes v_{3} \otimes v_{3}$ and $1_{A}$, the three properties of Definition 2.1 are readily seen to hold.

(2) The next example shows that the definition of a cellularly stratified algebra depends on the choice of the inflation data.

(a) Let $J$ be the inflation of the group algebra of the symmetric group $\Sigma_{2}=\left\langle\sigma: \sigma^{2}=\epsilon\right\rangle$ by the two-dimensional vector space $V$ with basis $v_{1}, v_{2}$. The multiplication in $J$ is governed by the bilinear form $\varphi$, where $\varphi\left(v_{i}, v_{j}\right)=\sigma$ if $i \neq j$ and zero otherwise. We form the algebra $A$ by adjoining an identity element to $J$. So $A$ is a nine-dimensional algebra. We define an involution $i$ by $i\left(\lambda \cdot 1_{A}+b \otimes u \otimes w\right)=\lambda \cdot 1_{A}+b \otimes w \otimes u$ for $\lambda \in k$, $b \in k \Sigma_{2}$ and $u, w \in V$. Note that this involution is compatible with the chosen bilinear form $\varphi$. Let $B=k \Sigma_{2}$. Assume that $e=1_{B} \otimes u \otimes w$ is an idempotent in $J$. Then

$$
e^{2}=(1 \otimes u \otimes w)(1 \otimes u \otimes w)=\varphi(w, u) \otimes u \otimes w .
$$

Let $u=\alpha v_{1}+\beta v_{2}$ and $w=\lambda v_{1}+\mu v_{2}$ for some $\alpha, \beta, \lambda, \mu \in k$. Then

$$
\varphi(w, u)=(\alpha \mu+\beta \lambda) \cdot \sigma \in k \cdot \sigma .
$$


Hence $\varphi(w, u) \neq 1_{B}$. So there are no idempotents in $J$ of the form $1_{k \Sigma_{2}} \otimes u \otimes w$, so $A$ does not satisfy Definition 2.1 of a cellularly stratified algebra with stratification data $\left(k \Sigma_{2}, V, k, k\right)$.

(b) We will verify that there is actually another inflation data that constructs an isomorphic copy of the algebra $A$. As before, let $J$ be the inflation of the group algebra of the symmetric group $\Sigma_{2}=\left\langle\sigma: \sigma^{2}=\epsilon\right\rangle$ by the two-dimensional vector space $V$ with basis $v_{1}, v_{2}$, and form $A$ by adjoining an identity element. This time we chose $\varphi\left(v_{i}, v_{j}\right)=\epsilon$ if $i \neq j$ and zero otherwise. This choice of bilinear form is conjugate to $\varphi$ chosen in part (a), and hence we obtain an algebra structure on $A$ which is isomorphic via a change of basis to the algebra structure considered in part (a). We define $e=e_{1}=\epsilon \otimes v_{1} \otimes v_{2}$ and $e_{2}=1_{A}$. Then property $(\mathrm{G})$ is seen to hold. We find $A e A=J$ and $e A e=\operatorname{span}_{k}\left\{\epsilon \otimes v_{1} \otimes v_{2}, \sigma \otimes v_{1} \otimes v_{2}\right\}$. We let $p=\left(\epsilon \otimes v_{1} \otimes v_{1}\right)$ and $q=\left(\epsilon \otimes v_{2} \otimes v_{2}\right)$. Then $e=p q$ and $i(e)=q p$ and $i(p)=p$ and the induced involution we obtain on $e A e$ is again the identity, so property $\left(\mathrm{J}^{\prime}\right)$ is satisfied. Finally we consider $A e$ as a right $e A e$-module:

$$
A e=\left\langle\epsilon \otimes v_{1} \otimes v_{2}, \sigma \otimes v_{1} \otimes v_{2}\right\rangle \oplus\left\langle\epsilon \otimes v_{2} \otimes v_{2}, \sigma \otimes v_{2} \otimes v_{2}\right\rangle .
$$

The first summand is $e A e$ and the second is qeAe, but both are isomorphic to $e A e$ as right $e A e$-modules and their intersection is trivial, thus property $\left(\mathrm{F}^{\prime}\right)$ is satisfied too. By Proposition 5.2, $A$ is a cellularly stratified algebra.

\section{Comparing Decomposition numbers}

Decomposition numbers of cellular algebras are by definition the multiplicities of simple modules $L$ as composition factors of cell modules $\Theta$. Given a cellular algebra $C$, denote its decomposition matrix by $D_{C}=\left(\left[\Theta_{C}(\lambda): L_{C}(\mu)\right]\right)_{\lambda, \mu}$ with cell modules $\Theta_{C}$ and simple modules $L_{C}$ and labels $\lambda \in \Lambda_{C}$ and $\mu \in \Lambda_{C}^{\text {simple }}$.

Proposition 6.1. Let $A$ be cellular and $e \in A$ be an idempotent. If the idempotent $e$ is fixed by the involution $i$, then eAe is also cellular, and $D_{e A e}$ is a diagonal submatrix of $D_{A}$. If $I$ is in the cell chain of $A$ then $A / I$ is again cellular, and $D_{A / I}$ is a diagonal submatrix of $D_{A}$.

Proof. The claim follows from the definition of cellular algebras by cell chains (see $[9,17,18]$ for instance), and from general theory as in Green [10, Section 6.2]: Assume the cell modules of $A$ are indexed by the elements in $\Lambda_{A}$. Then, as $i(e)=e, e A e$ is cellular and the cell chain of $e A e$ is obtained from that of $A$ by multiplying the cell chain of $A$ by $e$ from the left and the right. Modules of $A$ become $e A e$-modules by multiplying with $e$ from the left but some of these $A$-modules may become zero. In particular, multiplication by $e$ sends cell modules to cell modules or zero, and simples to simples or zero. That is, the cell modules and simple modules are

$$
\begin{aligned}
\Theta_{e A e}(\lambda)=e \Theta_{A}(\lambda) & \text { with } \lambda \in \Lambda_{e A e} \subseteq \Lambda_{A}, \\
L_{e A e}(\mu)=e L_{A}(\mu) & \text { with } \mu \in \Lambda_{e A e}^{\text {simple }} \subseteq \Lambda_{A}^{\text {simple }} .
\end{aligned}
$$

Hence $D_{e A e}$ is a diagonal submatrix of $D_{A}$. Similarly, if $I=I_{l}$ is an ideal in the cell chain of $A$, say

$$
A=I_{t} \supseteq I_{t-1} \supseteq \ldots \supseteq I_{0}=\{0\}
$$


then the cell chain of $A / I$ is given by

$$
A / I=I_{t} / I \supseteq I_{t-1} / I \supseteq \ldots \supseteq I_{l} / I=\{0\},
$$

and the cell modules and simple modules of the quotient algebra are

$$
\begin{array}{ll}
\Theta_{A / I}(\lambda)=\Theta_{A}(\lambda) & \text { with } \lambda \in \Lambda_{A / I} \subseteq \Lambda_{A}, \\
L_{A / I}(\mu)=L_{A}(\mu) & \text { with } \mu \in \Lambda_{A / I}^{\text {simple }} \subseteq \Lambda_{A}^{\text {simple }} .
\end{array}
$$

Hence $D_{A / I}$ is a diagonal submatrix of $D_{A}$.

Corollary 6.2. Let $A$ be cellularly stratified and assume that for each $l$ the idempotent $e=e_{l}$ is fixed by the involution $i$. Then the decomposition matrix of A contains on its diagonal precisely the decomposition matrices $D_{B_{l}}$ for $l=1, \ldots, n$. Moreover the decomposition matrices $D_{e A e}$ are submatrices of the right-hand bottom corner, the decomposition matrices $D_{A / J_{l}}$ are submatrices of the left-hand top corner as in the following picture:

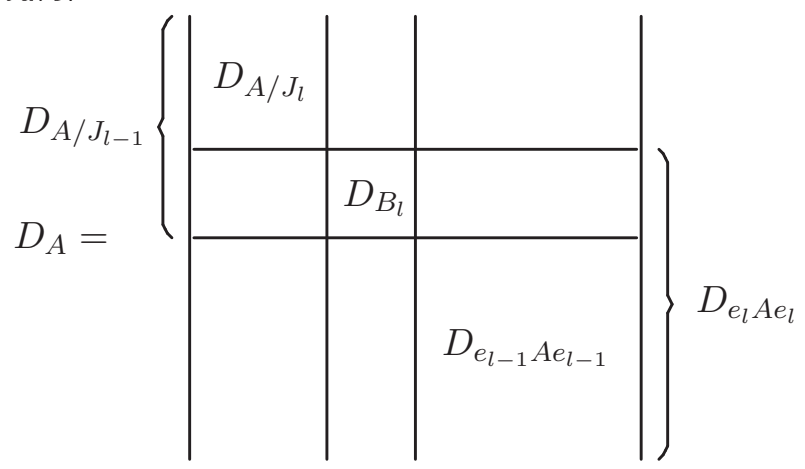

Proof. Note since $i(e)=e$ this implies $u=v$. Observe that $e\left(A / J_{l-1}\right) e \simeq B \otimes u \otimes u \simeq$ $B$, and then the result follows from Proposition 6.1.

Remark. For our main examples in this article - Brauer algebras, BMW algebras and partition algebras - in the case where the parameter $\delta \neq 0$ we have idempotents $e_{l}$ of the form $\lambda \cdot(1 \otimes u \otimes u)$ where $\lambda$ is a scalar, clearly satisfying $i\left(e_{l}\right)=e_{l}$. Hence the last corollary implies equalities between certain decomposition numbers of these algebras and decomposition numbers of group algebras of symmetric groups or their Hecke algebras (which are the algebras $B_{l}$ in these examples). In addition, since each $e A e$ is isomorphic to a smaller diagram algebra of the same type we see, for example, that the decomposition matrix of $B_{k}(r-2, \delta)$ (respectively $B M W_{k}\left(r-2, \lambda, q-q^{-1}, \delta\right.$ ) or $P_{k}(r-1, \delta)$ ) is included in that of $B_{k}(r, \delta)$ (respectively $B M W_{k}\left(r, \lambda, q-q^{-1}, \delta\right)$, or $\left.P_{k}(r, \delta)\right)$.

However the idempotents $e_{l}$ for a cellularly stratified algebra may be of the form $1 \otimes u \otimes v$ with $u \neq v$, and then $i\left(e_{l}\right) \neq e_{l}$. In certain such cases we still obtain the above results relating decomposition numbers. Under the additional assumption that the idempotents $e_{l}$ and $i\left(e_{l}\right)$ are orthogonal, $\tilde{e}_{l}=e_{l}+i\left(e_{l}\right)$ is an idempotent of $A$ and we may replace $e_{l}$ by $\tilde{e}_{l}=e_{l}+i\left(e_{l}\right)$ in the proof of the corollary. Then $\tilde{e}_{l}\left(A / J_{l-1}\right) \tilde{e}_{l}$ is the inflation $B \otimes \operatorname{span}\{u, v\} \otimes \operatorname{span}\{u, v\}$, of $B$, and since the bilinear form for this 
inflation is non-singular, the decomposition matrices of $\tilde{e}_{l}\left(A / J_{l-1}\right) \tilde{e}_{l}$ and of $B$ coincide, see [21, Corollary 3.4]. Such a situation occurs for the Brauer and BMW algebras when $r$ is odd and $\delta=0$.

\section{Cellularly stratified algebras are stratified}

One of our aims is to extend the phenomenon discovered by Hemmer and Nakano [13] to certain diagram algebras. This phenomenon identifies homomorphism spaces and it identifies first extension groups (for the case of symmetric groups and their quantizations, see [13], Theorem 3.7.1 and Corollary 3.9.1). However, it identifies higher extensions only with higher relative extensions. For cellularly stratified algebras there is another homological structure. In this section we provide a stratification of the derived module category of any cellularly stratified algebra, independent of the HemmerNakano phenomenon. We start by recalling what stratified algebras are. There are various, in fact non-equivalent, definitions of stratified algebras. Here we follow the most general of these definitions, due to Cline, Parshall, Scott [3, 2.1.1].

Definition 7.1. An algebra $A$ is stratified if there exists a chain of ideals $\{0\}=J_{0} \subseteq$ $J_{1} \subseteq \ldots \subseteq J_{n-1} \subseteq J_{n}=A$ such that each subquotient $J_{i} / J_{i-1}$ is a stratifying ideal in the quotient algebra $A / J_{i-1}$. Here an ideal $J$ in an algebra $A$ is called a stratifying ideal provided that the following conditions hold:

(1) There is an idempotent $e \in A$ such that $J=A e A$.

(2) Multiplication provides an $A$-bimodule isomorphism $A e \otimes_{e A e} e A \rightarrow J$.

(3) $\operatorname{Tor}_{n}^{e A e}(A e, e A)=0$ for all $n>0$.

Remark. An equivalent way to phrase conditions (1) to (3) is to require that the derived functor $D^{+}(A / J$-mod $) \rightarrow D^{+}(A$-mod $)$ induced by the full embedding $A / J-\bmod \rightarrow$ $A-\bmod$ is a full embedding, see $[3,2.1 .2]$.

Assume from now on that $A$ is cellularly stratified. Next we show that the lowest layer in the chain of ideals provided by condition (C) is a stratifying ideal. As a consequence cellularly stratified algebras are stratified.

Proposition 7.2. Suppose the algebra $A$ is cellularly stratified, with notation as above. Then $A$ is stratified with a stratification provided by the ideals $J_{l}$.

Proof. We have to show that $J_{1}$ is a stratifying ideal in $A, J_{2} / J_{1}$ is a stratifying ideal in $A / J_{1}$, and so on. We do this by induction on the layers. Write $e=e_{1}, B=B_{1}$ and $V=V_{1}$. By the assumptions on $A$, the lowest layer is the ideal $J=A e A=B \otimes V \otimes V$, and $B \simeq e A e$.

By Proposition 3.5, the right module $A e=B \otimes V \otimes v$ - and similarly the left module $e A=B \otimes u \otimes V$ - is a free $B$-module of rank $\operatorname{dim}_{k} V$. Hence $A e$ is flat, and the third condition for a stratifying ideal holds: $\operatorname{Tor}_{n}^{e A e}(A e, e A)=0$ for all $n>0$. The map

$$
A e \otimes_{e A e} e A \rightarrow J=B \otimes V \otimes V
$$


given by multiplication is an isomorphism of vector spaces (see Equation (3) from Section 2). This shows that $J$ is a stratifying ideal in $A$. The claim now follows by induction on the layers.

Remark. In the case of the Brauer algebra $B_{k}(r, \delta)$ with $\delta=0$ and $r$ even, the ideal spanned by totally horizontal diagrams is not a stratifying ideal. However in this case, the previous proposition is true for the quotient of the Brauer algebra obtained by factoring out this ideal.

In general, a stratifying ideal induces only partial recollement diagrams, see [3, Section 2.1.2] and [16], where definitions of recollements can also be found. Here we obtain more:

Theorem 7.3. Assume that $A$ is cellularly stratified.

(a) Then there is a full recollement of bounded derived categories

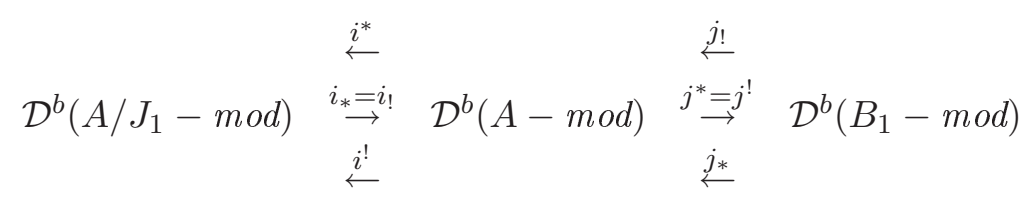

(b) The derived category of $A$ has a stratification (iterated recollements) by the derived categories of the algebras $B_{l}$.

Proof. (a) We write $e=e_{1}, J=J_{1}$ and $B=B_{1}$, and so by Lemma 2.3, $B \simeq e A e$. It is clear that $\operatorname{Hom}_{A}(A e A, A / J)=0$, and that $A e A \simeq \bigoplus A e$ is projective as an $A$-module. So we can apply [16, Corollary 12] and obtain a full recollement for $\mathcal{D}^{-}$as above. We can replace $\operatorname{End}_{A}(A e A)$ by $e A e \simeq B$, since, as a left $A$-module $A e A$ is just a sum of copies of the projective $A$-module $A e$, so that the two algebras are Morita equivalent. Now [16, Lemma 2] implies that this recollement restricts to a right recollement for the bounded derived categories, that is, the bottom four functors take complexes with bounded homology to complexes with bounded homology. It is left to show that the upper two functors do that as well. Note that all the six occurring functors are induced by functors on the corresponding module categories (denoted by the same symbols), namely

$$
\begin{aligned}
i_{*}=i_{!}:={ }_{A}(A / J) \otimes_{A / J}-: & A / J \text {-mod } \rightarrow A \text {-mod }, \\
i^{*}:=A / J \otimes_{A}-: & A \text {-mod } \rightarrow A / J \text {-mod } \\
i^{!}:=\operatorname{Hom}_{A}(A / J,-): & A \text {-mod } \rightarrow A / J \text {-mod } \\
j^{*}=j^{!}:=e A \otimes_{A}-: & A \text {-mod } \rightarrow e A e \text {-mod, } \\
j_{!}:=A e \otimes_{e A e}-: & e A e-\bmod \rightarrow A \text {-mod }, \\
j_{*}:=\operatorname{Hom}_{e A e}(e A,-): & e A e-\bmod \rightarrow A \text {-mod }
\end{aligned}
$$

Here $A e$ is a projective right $e A e$-module, hence flat, therefore $\operatorname{Tor}_{j}^{e A e}(A e,-)=0$ for $j \geq 1$. So the derived functor of $j$ ! takes complexes with bounded homology to complexes with bounded homology. In $\mathcal{D}^{b}(A)$, the module $A / J$ is isomorphic to the complex $Y:=(\cdots \rightarrow 0 \rightarrow A e A \rightarrow A \rightarrow 0 \rightarrow \ldots)$ of projective (hence flat) $A$-modules. 
Let $X \in \mathcal{D}^{b}(A-\bmod )$. The homology of $i^{*}(X)$ is just the homology of the total complex $T$ of the double complex $Y \otimes_{A} X$, which vanishes in high degrees, since the homology of $X$ is zero for high degrees, and tensoring with flat modules is exact. Hence $i^{*}(X) \in \mathcal{D}^{b}(A / J-\bmod )$, as required.

(b) Replacing $A / J_{l-1}$ by $A / J_{l}$ and $e=e_{l}$ by the idempotent $e_{l+1}$ in the next layer and iterating the above argument, we obtain a sequence of recollement diagrams, each of them having the derived category of the respective $B_{l}$-mod on the right hand side. In the last step, we have $A / J_{n-1}$ on the left hand side, which is isomorphic to the algebra $B_{n}$ since $e_{n}=1$. The claim follows.

The last theorem has various consequences. In particular, using [3, Section 2.1.2], we obtain a comparison of Ext-groups:

Corollary 7.4. Let $A$ be cellularly stratified. Let $M, N$ be any $A / J_{l}$-modules and $X, Y$ any $B_{l}$-modules. Then for any $i>0$ and any $j \geq 0$ we have:

$$
\begin{aligned}
& \operatorname{Ext}_{A}^{i}(M, N) \simeq \operatorname{Ext}_{A / J_{l}}^{i}(M, N), \\
& \operatorname{Ext}_{B_{l}}^{j}(X, Y) \simeq \operatorname{Ext}_{A}^{j}\left(G_{l}(X), G_{l}(Y)\right) .
\end{aligned}
$$

Proof. The first isomorphism follows from [3, Section 2.1.2]. The case $j=0$ of the second isomorphism has been obtained in Proposition 4.3. In the case $j>0$, we apply the first isomorphism to obtain

$$
\operatorname{Ext}_{A}^{j}\left(G_{l}(X), G_{l}(Y)\right) \simeq \operatorname{Ext}_{A / J_{l-1}}^{j}\left(G_{l}(X), G_{l}(Y)\right) .
$$

Using the definition of the functors $G_{l}$ (see Section 3), this equals

$$
\operatorname{Ext}_{A / J_{l-1}}^{j}\left(\left(A / J_{l-1}\right) e_{l} \otimes_{e_{l}\left(A / J_{l-1}\right) e_{l}} X,\left(A / J_{l-1}\right) e_{l} \otimes_{e_{l}\left(A / J_{l-1}\right) e_{l}} Y\right) \text {. }
$$

Since $e_{l}$ is in the lowest layer of $A / J_{l-1}$, it follows that $e_{l}\left(A / J_{l-1}\right) e_{l} \simeq B_{l}$. We now apply Theorem 7.3 to the cellularly stratified algebra $A / J_{l-1}$. Then

$$
\operatorname{Ext}_{A}^{j}\left(G_{l}(X), G_{l}(Y)\right) \simeq \operatorname{Ext}_{A / J_{l-1}}^{j}\left(j_{!}(X), j_{!}(Y)\right) .
$$

Since $j !: \mathcal{D}^{b}\left(B_{l}-\bmod \right) \rightarrow \mathcal{D}^{b}\left(A / J_{l-1}-\bmod \right)$ is a full embedding, it follows that

$$
\operatorname{Ext}_{A}^{j}\left(G_{l}(X), G_{l}(Y)\right) \simeq \operatorname{Ext}_{B_{l}}^{j}(X, Y) .
$$

Let $A$ be any algebra. Recall that the projective dimension of an $A$-module $M$ is the length of a minimal projective resolution of $M$. The global dimension of the algebra $A$ is then the maximum of the projective dimensions of the $A$-modules. For many algebras this number will not be finite. In those cases one also considers the finitistic dimension. The finitistic dimension of an algebra $A$ is the maximum of the projective dimensions of all those $A$-modules which have a finite projective resolution. It has been conjectured that the finitistic dimension is always finite. A positive answer to this conjecture for finite dimensional algebras would imply validity of various other conjectures such as the Nakayama conjecture. For more information see the surveys given in [8, 26, 27]. In 
the case of cellularly stratified algebras $A$, we can reduce the question of finiteness of the finitistic dimension to the same question for the smaller algebras $B_{l}$ :

Corollary 7.5. Let $A$ be cellularly stratified by the algebras $B_{1}, \ldots, B_{n}$. Then the global dimension of $A$ is finite if and only if all the algebras $B_{l}$ have finite global dimensions. The finitistic dimension of $A$ is finite if and only if all the algebras $B_{l}$ have finite finitistic dimensions.

Proof. The first claim follows from Theorem 7.3 together with [16, Corollary 5]. The second claim follows from Theorem 7.3 together with [11, Theorem 2].

Corollary 7.6. The finitistic dimension conjecture holds for Brauer algebras (with $\delta \neq 0$ if $r$ is even), $B M W$-algebras (with $\delta \neq 0$ if $r$ is even) and partition algebras (with $\delta \neq 0)$.

Proof. Here the algebras $B_{l}$ are group algebras of symmetric group or their Hecke algebras which are self-injective algebras. Self-injective algebras are easily seen to have finitistic dimension zero: Assume that a finite projective resolution of a non-projective module $M$ is given. In a self-injective algebra projective modules are injective. Hence the finite exact sequence of the projective resolution of $M$ splits in the leftmost term, a contradiction. This implies that a module for any self-injective algebra is either projective or has no finite projective resolution. The statement now follows from Corollary 7.5 .

Remark. Working in a more general context, Frisk proved several results on when the finitistic dimension of a standardly stratified algebra is finite. He also gives an upper bound for the finitistic dimension, depending on the finitistic dimension of the endomorphism algebras of standard modules, see for example [7, Theorem 24].

\section{Homomorphisms and EXtensions BetWeEn LAYERS}

We have seen in Proposition 4.3 and Corollary 7.4 that homomorphisms and extensions of cell modules of $A$ inside the $l$ th layer behave like those of the small algebra $B_{l}$. In this section we will study homomorphisms and extensions between cell modules of different layers. We will see that homomorphisms and extensions between cell modules can happen either in the same layer or from a higher to a lower layer, but not the other way round.

Proposition 8.1. Let $l<m$, and let $X$ be a $B_{m}$-module and $Y$ a $B_{l}$-module. Then

$$
\operatorname{Hom}_{A}\left(A e_{l} \otimes_{e_{l} A e_{l}} B_{l} \otimes_{B_{l}} Y, A e_{m} \otimes_{e_{m} A e_{m}} B_{m} \otimes_{B_{m}} X\right)=0 .
$$

In particular, if $\Theta(\lambda)$ and $\Theta(\mu)$ are cell modules, with $\Theta(\lambda)$ in the layer of $e_{l}$ and $\Theta(\mu)$ in the layer of $e_{m}$ with $l<m$, then $\operatorname{Hom}_{A}(\Theta(\lambda), \Theta(\mu))=0$.

Proof. Using Proposition 4.2, we can write $\Theta(\mu)=A e_{m} \otimes_{e_{m} A e_{m}} B_{m} \otimes_{B_{m}} X$, where $X$ is a cell module for $B_{m}$ and similarly, $\Theta(\lambda)=A e_{l} \otimes_{e_{l} A e_{l}} B_{l} \otimes_{B_{l}} Y$ where $Y$ is a cell module for $B_{l}$. Hence it suffices to prove the first claim. 
Using the adjointness of the Hom-functor and the tensor functor, and using assumption (I), we then have:

$$
\begin{aligned}
& \operatorname{Hom}_{A}\left(A e_{l} \otimes_{e_{l} A e_{l}} B_{l} \otimes_{B_{l}} Y, A e_{m} \otimes_{e_{m} A e_{m}} B_{m} \otimes_{B_{m}} X\right) \\
= & \operatorname{Hom}_{A}\left(A e_{l} \otimes_{e_{l} A e_{l}} Y, A e_{m} \otimes_{e_{m} A e_{m}} X\right) \\
\simeq & \operatorname{Hom}_{e_{l} A e_{l}}\left(Y, \operatorname{Hom}_{A}\left(A e_{l}, A e_{m} \otimes_{e_{m} A e_{m}} X\right)\right) \\
= & \operatorname{Hom}_{e_{l} A e_{l}}\left(Y, e_{l} A e_{m} \otimes_{e_{m} A e_{m}} X\right) \\
= & \operatorname{Hom}_{e_{l} A e_{l}}\left(Y, e_{l} X\right)=\operatorname{Hom}_{e_{l} A e_{l}}(Y, 0)=0 .
\end{aligned}
$$

Note that $X$ is a $B_{m}$-module, and as $e_{m} A e_{m}$ surjects onto $B_{m}$, it is also an $e_{m} A e_{m^{-}}$ module with $e_{m} J_{m-1} e_{m} \cdot X=0$. So in particular $e_{l} X=0$.

Similarly, extensions between cell modules can happen either in the same layer or from a higher to a lower layer but not the other way round; this resembles the situation for quasi-hereditary algebras where each layer has just one index and one simple module.

Proposition 8.2. Let $l<m$, and let $X$ be a $B_{l}$-module and $Y$ a $B_{m}$-module. Then for all $i \geq 1$,

$$
\operatorname{Ext}_{A}^{i}\left(A e_{l} \otimes_{e_{l} A e_{l}} B_{l} \otimes_{B_{l}} Y, A e_{m} \otimes_{e_{m} A e_{m}} B_{m} \otimes_{B_{m}} X\right)=0 .
$$

In particular, if $\Theta(\lambda)$ and $\Theta(\mu)$ are cell modules, with $\Theta(\lambda)$ in the layer of $e_{l}$ and $\Theta(\mu)$ in the layer of $e_{m}$ with $l<m$, then $\operatorname{Ext}_{A}^{i}(\Theta(\lambda), \Theta(\mu))=0$ for all $i \geq 1$.

Proof. As before, it suffices to prove the more general first claim. For the $B_{m^{-}}$ module $X$ and $B_{l}$-module $Y$, we have $G_{m}(X)=A e_{m} \otimes_{e_{m} A e_{m}} B_{m} \otimes_{B_{m}} X$ and $G_{l}(Y)=$ $A e_{l} \otimes_{e_{l} A e_{l}} B_{l} \otimes_{B_{l}} Y$. Since $J_{l} \cdot G_{m}(X)=0$ and as $e_{l} \in J_{l}$, this implies

$$
\operatorname{Hom}_{A}\left(A e_{l}, G_{m}(X)\right)=e_{l} \cdot G_{m}(X)=0 .
$$

Consider the lowest layer, that is $B_{l}=B_{1}$. Here we have $e_{l} A e_{l} \simeq B_{l}$ and $A e_{l}$ is a projective right $e_{l} A e_{l}$-module (see Proposition 3.5). The induction functor $G$ sends $B_{l}$ to the projective $A$-module $A e_{l}$. The functor $G$ is exact by Proposition 4.1, hence it sends a $B_{l}$-projective resolution of $Y$ to an $A$-projective resolution of $G_{l}(Y)$, say

$$
\ldots \rightarrow P_{1} \rightarrow P_{0} \rightarrow G_{l}(Y) \rightarrow 0
$$

where $P_{0}, P_{1}, \ldots$ are direct summands of $\bigoplus A e_{l}$. But by Equation (8), it follows that $\operatorname{Hom}_{A}\left(P_{i}, G_{m}(X)\right)=0$ for all $i$, so by the definition of Ext ${ }^{i}$, this shows the claim in the lowest layer. For layers $l$ and $m$ with $l<m$ consider $A / J_{l-1}$. By (6), the $A / J_{l-1}$-module $\bar{G}_{l}(Y)$, viewed as an $A$-module, is isomorphic to $G_{l}(Y)$. Thus $\operatorname{Ext}_{A}^{i}\left(G_{l}(Y), G_{m}(X)\right) \simeq$ $\operatorname{Ext}_{A / J_{l-1}}^{i}\left(G_{l}(Y), G_{m}(X)\right)=0$ by the above, using Corollary 7.4.

\section{Comparing Cellular and stratified Algebras on EXamples}

Cellular algebras have often been considered as a combinatorial generalization of quasihereditary algebras while stratified algebras have been considered as a homological one. We have seen in Section 7 that there are cellular algebras which are stratified. In this section, we illustrate by an example that the Ext-comparison (see Corollary 7.4) and 
Ext-vanishing properties (see Proposition 8.2) of stratified algebras do not hold for cellular algebras in general. We consider the algebra $A=k Q / R$ given by the quiver

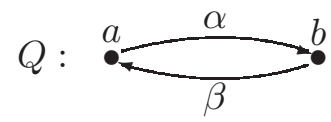

with relations $R=\langle\alpha \beta \alpha, \beta \alpha \beta\rangle$. Then $A$ is a six dimensional algebra with basis $\{a, b, \alpha, \beta, \alpha \beta, \beta \alpha\}$. Define a map $i$ with

$$
i(\alpha)=\beta, \quad i(\beta)=\alpha, \quad i(a)=a, \quad i(b)=b,
$$

and extend it anti-multiplicatively. Then the algebra $A$ is cellular with involution $i$ and cell chain $A=J_{3} \supseteq J_{2} \supseteq J_{1} \supseteq J_{0}=\{0\}$ where the ideals are given as follows:

$$
J_{2}=A a A=\operatorname{span}_{k}\{a, \alpha, \beta, \alpha \beta, \beta \alpha\}, \quad J_{1}=\langle\alpha \beta\rangle=\operatorname{span}_{k}\{\alpha \beta\} .
$$

Then the quotient algebras $A / J_{i}$ for $1 \leq i \leq 3$ have the following decompositions into projective indecomposable modules:

$$
A=\begin{array}{ccc}
a & b \\
b & \oplus & a \\
a & b
\end{array}, \quad A / J_{1}=\begin{aligned}
& a \\
& b
\end{aligned} \oplus \begin{aligned}
& b \\
& a \\
& b
\end{aligned} \quad \text { and } \quad A / J_{2}=b .
$$

Here $a$ and $b$ denote the two simple one-dimensional $A$-modules. The cell modules are

$$
\Theta_{1}=a, \quad \Theta_{2}=\begin{aligned}
& a \\
& b
\end{aligned}, \quad \Theta_{3}=b .
$$

Here, for instance, $J_{1}$ as a left $A$-module equals $a$ and $J_{1}$ is isomorphic to $\Theta_{1} \otimes_{k} i\left(\Theta_{1}\right)$ via multiplication so $J_{1}=J_{1} / J_{0}$ is a cell ideal in $A=A / J_{0}$. As there are uniserial $A$-modules $[a, b]$ and $[b, a]$, we have non-split extensions of the cell module $\Theta_{1}$ with $\Theta_{3}$ and vice versa, and hence Proposition 8.2 does not hold for $A$.

A minimal projective resolution of the $A$-module $a$ is given by the following complex which is periodic of length four:

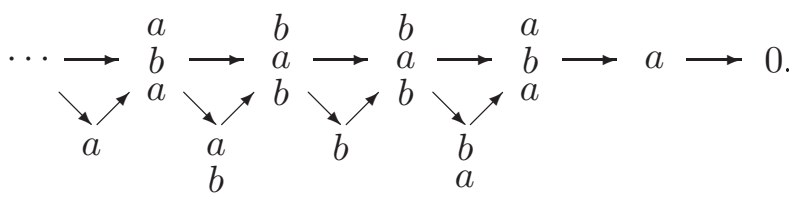

The same complex can be used to read off the minimal projective resolution of $b$ over $A$. As an $A / J_{1}$-module a minimal projective resolution of $a$ is given by the following finite complex, which also can be used to read off the minimal projective resolution of $b$ :

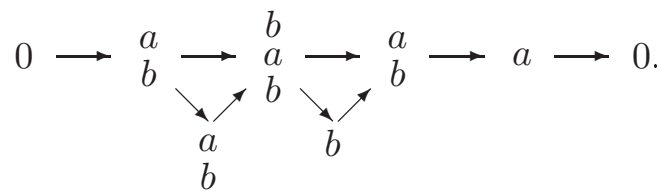

Applying $\operatorname{Hom}_{A}(-, a)$ and $\operatorname{Hom}_{A / J_{1}}(-, a)$ respectively to these complexes, we can calculate the extension groups. For instance, we obtain that $\operatorname{Ext}_{A / J_{1}}^{m}(a, a)=0$ for all $m \geq 3$, but $\operatorname{Ext}_{A}^{4 t}(a, a)=\operatorname{Ext}_{A}^{4 t+3}(a, a)=k$ for $t \geq 0$. So, for $A$ a general cellular algebra, we cannot identify higher extension groups for $A$ with those for $A / J$. 
Using a different ideal chain that avoids nilpotent layers does not improve the situation: The ideal $J_{1}$ is nilpotent, and there are no simple $A$-modules corresponding to nilpotent layers of the cell chain of $A$. Thus we now try the ideal chain $A \supset J_{2} \supset\{0\}$ which also refines to the cell chain of $A$ given above. Then neither $J_{2}$ as an ideal of $A$ nor $A / J_{2}$ as an ideal of itself are nilpotent ideals. However, this is still not enough to obtain a stratification, the ideal $J_{2}$ fails to be stratifying. As one easily calculates, the first two conditions of Definition 7.1 hold, but the third one does not. To see this, note that the algebra $C:=a A a$ is two-dimensional, it has one simple module $a$ and $C$ is the unique indecomposable projective. The (right) $C$-module $A a \simeq a \oplus C$ has the projective resolution

$$
\cdots \rightarrow C \rightarrow C \rightarrow C \oplus C \rightarrow a \oplus C \rightarrow 0
$$

and tensoring with $a A \simeq a \oplus C$ (from the right) gives a complex with non-zero homology in all degrees, hence $\operatorname{Tor}_{n}^{C}(A a, a A) \neq 0$ for all $n$. So the absence of nilpotent ideals in the chain is not enough, we need cellularity and the idempotents, as in Definition 2.1.

\section{Cellularly stratified algebras and standard systems}

Using the technology set up in the previous sections, we are now ready to state the Hemmer-Nakano phenomenon for cellularly stratified algebras, exhibiting the algebraic Lie theory hidden in the diagram algebras studied here. Recall the notion of a standardizable set by Dlab and Ringel [5, Section 3] - here called a standard system - of objects in an abelian category, given here for a module category:

Definition 10.1. Let $C$ be any algebra, and suppose we are given a finite set $\Theta$ of non-isomorphic $C$-modules $\Theta(j)$, indexed by $j \in I$, where $I$ is endowed with a partial order $\leq$. Then the modules $\Theta(j)$ are said to form a standard system if the following three conditions hold:

(i) For all $j \in I, \operatorname{End}_{C}(\Theta(j))$ is a division ring.

(ii) For all $m, n \in I$, if $\operatorname{Hom}_{C}(\Theta(m), \Theta(n)) \neq 0$ then $m \geq n$.

(iii) For all $m, n \in I$, if $\operatorname{Ext}_{C}^{1}(\Theta(m), \Theta(n)) \neq 0$ then $m>n$.

\section{Remarks.}

(1) In our examples of cellularly stratified algebras $A$ with algebras $B_{l}$ being group algebras of symmetric groups, if $k$ is a field of characteristic not equal to two then the Specht modules have one-dimensional endomorphism rings [15, Corollary 13.17], and then Propositions 4.2 and 4.3 imply condition (i) for the set of cell modules.

(2) In a standard system, condition (i) implies that all $\Theta(j)$ are indecomposable, a property that is not in general shared by the cell modules of an arbitrary cellular algebra.

(3) The partial order used above can be refined, for example into a total order, and trivially (i)-(iii) hold for the refined order.

(4) If the cell modules of a cellular algebra form a standard system then the dual cell modules also form a standard system with respect to the dual order. 
It is well known that $\Delta$-filtration multiplicities of modules over quasi-hereditary algebras are well-defined (see [2]). More precisely, let $A$ be a quasi-hereditary algebra. Denote by $\mathcal{F}(\Delta)$ the category of modules with a standard filtration ( $\Delta$-filtration), and let $X \in \mathcal{F}(\Delta)$. Then the number of times a particular module $\Delta(j)$ occurs as a subquotient in a $\Delta$-filtration of $X$ is independent of the filtration chosen. We sketch an argument for this: Let $X \in \mathcal{F}(\Delta)$. Denote costandard modules, which occur in the filtrations of injective modules, by $\nabla(j)$. Take a $\Delta$-filtration of the module $X$, say

$$
0=X_{0} \subseteq X_{1} \subseteq \ldots \subseteq X_{t}=X,
$$

and denote by $[X: \Delta(j)]$ the multiplicity of $\Delta(j)$ occurring in this filtration of $X$. We inductively determine $\left[X / X_{i}: \Delta(j)\right]$ for $i=t, t-1, \ldots, 0$. Apply the functor $\operatorname{Hom}_{A}(-, \nabla(j))$ to the short exact sequences

$$
0 \rightarrow X_{i+1} / X_{i} \rightarrow X / X_{i} \rightarrow X / X_{i+1} \rightarrow 0
$$

with $0 \leq i \leq t-2$. Note that $\operatorname{Hom}_{A}(-, \nabla(j))$ is exact on $\mathcal{F}(\Delta)$ since $\operatorname{Ext}_{A}^{i}(\Delta(l), \nabla(j))=0$ for $j, l \in I, i \geq 1$. Hence

$$
\left[X / X_{i}: \Delta(j)\right]=\left[X_{i+1} / X_{i}: \Delta(j)\right]+\left[X / X_{i+1}: \Delta(j)\right]
$$

for every $i$. Moreover, $\operatorname{Hom}_{A}(\Delta(l), \nabla(j))=0$ unless $l=j$, in which case the Hom space is free of rank one over $\operatorname{End}_{A}(\Delta(j))=\operatorname{End}_{A}(\nabla(j))=\operatorname{End}_{A}(L(j))$. This implies that

$$
\operatorname{dim} \operatorname{Hom}_{A}(X, \nabla(j)) / \operatorname{dim} \operatorname{End}_{A}(L(j))=[X: \Delta(j)] .
$$

Hence $[X: \Delta(j)]$ is independent of the chosen filtration of $X$.

Assume an algebra $C$ has a standard system $\Theta$. Denote by $\mathcal{F}(\Theta)$ the category of $C$ modules with a $\Theta$-filtration. Then by [5, Theorem 2], there exists a quasi-hereditary algebra $S(C)$ with index set $(I, \leq)$ and standard modules $\Delta$ such that $\mathcal{F}(\Delta) \simeq \mathcal{F}(\Theta)$ (as exact categories). Here $\mathcal{F}(\Delta)$ denotes the category of $S(C)$-modules with a $\Delta$ filtration. The equivalence sends the standard module $\Delta(j)$ to $\Theta(j)$, and hence modules with a standard filtration to modules with a cell filtration. Using the equivalence $\mathcal{F}(\Delta) \simeq \mathcal{F}(\Theta)$, this implies that any module $X \in \mathcal{F}(\Theta)$ has well-defined $\Theta$-filtration multiplicities.

Theorem 10.2. Let $A$ be cellularly stratified.

(a) Then the cell modules of $A$ form a standard system if and only if for each $l$ the cell modules of $B_{l}$ form a standard system.

(b) Assume that for each $l$ the cell modules of $B_{l}$ form a standard system. Then an A-module with a cell filtration has well-defined filtration multiplicities.

Proof. Combine Proposition 4.3 and Corollary 7.4 with Propositions 8.1 and 8.2.

Hemmer and Nakano have shown in [13, 4.2.1 and 4.4.1] that in case of $k$ having characteristic different from two or three, the Specht modules (with the dominance order) form a standard system for the group algebra of the symmetric group. Similarly, they show that for $e \geq 4$, where $e$ is least such that $1+q^{-2}+q^{-4}+\cdots+q^{-2 e}=0$, the Hecke algebra $\mathcal{H}_{l}\left(q^{-2}\right)$ has a standard system composed of Specht modules. We can combine Theorem 10.2 with the results in [13] to say that if $A$ is cellularly stratified with 
the cellular algebras $B_{l}$ being group algebras of symmetric groups (or Hecke algebras respectively) and the characteristic of $k$ is different from two and three (or $e \geq 4$ respectively), then $A$ has well-defined cell filtration multiplicities. Under some mild assumptions this is the case for the three main examples of this article:

Corollary 10.3. (a) Consider a Brauer algebra - with $\delta \neq 0$ in the case of $r$ even - or a partition algebra - with $\delta \neq 0$. Then its cell modules form a standard system if $\operatorname{char}(k) \neq 2,3$. In this case, modules with cell filtrations have well-defined filtration multiplicities.

(b) The cell modules of the BMW algebra - with $\delta \neq 0$ in the case of $r$ even - form a standard system if $e \geq 4$. In this case, modules with cell filtrations have well-defined filtration multiplicities.

Remark. Note that Propositions 8.1 and 8.2 need no assumptions, apart from $A$ being cellularly stratified. Thus, the assumptions needed in these corollaries are only used within the layers, not in between layers.

\section{The equivalence $\mathcal{F}(\Theta) \longrightarrow \mathcal{F}(\Delta)$ For cellular algebras}

The results of Dlab and Ringel [5, Section 3] are for a standard system $\Theta$ in any abelian category. Here we apply this theory, and provide additional detail, in the case of a cellular algebra whose cell modules form a standard system. So let $A$ be cellular with cell modules $\Theta(\lambda)$ for $\lambda$ in the index set $(\Lambda, \leq)$. Assume the cell modules of $A$ form a standard system. As mentioned in the previous section, by [5, Theorem 2], there exists a quasi-hereditary algebra $S(A)$ with index set $(\Lambda, \leq)$ and standard modules $\Delta$ such that $\mathcal{F}(\Delta) \simeq \mathcal{F}(\Theta)$ (as exact categories). The equivalence sends the standard module $\Delta(\lambda)$ to $\Theta(\lambda)$.

Remark. It is known for a quasi-hereditary algebra that the full subcategory of modules filtered by the standard modules of the algebra is closed under taking direct summands. Hence [5, Theorem 2] implies that $\mathcal{F}(\Theta)$ is closed under taking direct summands. By the definition of cellular algebras, $A$ is filtered by cell modules, and so $A$, and all its direct summands - that is, all projective $A$-modules - lie in $\mathcal{F}(\Theta)$.

Example 11.1 (see Proposition 7.1 of [17]). For a cellular algebra with cell modules $\Theta$, the category $\mathcal{F}(\Theta)$ is in general not closed under taking direct summands. To obtain an example, take an algebra $B$ which is cellular with involution $i$ and let $X$ be some $B$-module. Define $M=X \otimes_{k} i(X)$. Define $C$ to be the vector space $B \oplus M$ as a vector space and identify $C$ with the set of $2 \times 2$ matrices

$$
\left\{\left(a_{i j}\right) \mid a_{21}=0, a_{11}=a_{22} \in B \text { and } a_{12} \in M\right\} .
$$

and define a multiplication on $C$ via matrix multiplication. Then $C$ is a cellular algebra with $M$ a cell ideal in $C$ and $X$ a cell module of $C$. Choosing a decomposable module $X$ whose direct summands are not cell modules of $B$, provides examples of cellular algebras $C$ with $\mathcal{F}(\Theta)$ not closed under taking direct summands. For example, take as the algebra $B$ the algebra $A / J_{1}$ appearing in Section 9 , and let $X$ be the $B$-module $X=a \oplus a$. In this case $C$ has the cell modules

$$
\Theta_{1}=[a, b], \quad \Theta_{2}=b \quad \text { and } \quad \Theta_{3}=a \oplus a
$$


with $\Theta_{1}, \Theta_{2}$ uniserial. Here $\mathcal{F}(\Theta)$ does not contain the direct summand $a$ of $\Theta_{3}$.

Definition 11.2. Let $Y$ and $M$ be left $A$-modules filtered by cell modules. We say $Y$ is relative projective in $\mathcal{F}(\Theta)$ if $\operatorname{Ext}_{A}^{1}(Y, N)=0$ for any module $N \in \mathcal{F}(\Theta)$. Moreover, we say $Y$ is the relative projective cover of $M$ if

(1) $Y$ is relative projective;

(2) there is a surjection $\epsilon: Y \rightarrow M$ with $\operatorname{ker}(\epsilon) \in \mathcal{F}(\Theta)$;

(3) for any other relative projective $Y^{\prime} \in \mathcal{F}(\Theta)$ and any surjection $\epsilon^{\prime}: Y^{\prime} \rightarrow M$ with $\operatorname{ker}\left(\epsilon^{\prime}\right) \in \mathcal{F}(\Theta)$, there exists a map $f: Y^{\prime} \rightarrow Y$ such that $\epsilon^{\prime}=\epsilon \circ f$

Remark. The property of being a relative projective cover is preserved under exact equivalences.

Construction of the algebra $S(A)$. By [5, Section 3], the elements of the standard system $\{\Theta(\lambda)\}$ are in one-to-one correspondence with the indecomposable relative projective $A$-modules in the category $\mathcal{F}(\Theta)$ of $\Theta$-filtered $A$-modules. Let $\left\{Y_{p r}(\lambda)\right\}$ be the relative projective cover of $\Theta(\lambda)$; here $Y_{p r}(\lambda)$ is constructed using iterated universal extensions, for details see [5, Section 3].

Take $Y=\bigoplus Y_{p r}(\lambda)^{a_{\lambda}}$ where the sum runs through all indices $\lambda \in \Lambda$ and where $a_{\lambda}$ is chosen to equal the dimension of $L(\lambda)$ if such a simple $A$-module exists, or equals 1 otherwise. Then the quasi-hereditary algebra $S(A)$, defined in [5], corresponding to the cellularly stratified algebra $A$ is given by

$$
S(A)=\operatorname{End}_{A}(Y) .
$$

The functors realizing the equivalence $\mathcal{F}(\Theta) \longrightarrow \mathcal{F}(\Delta)$. The equivalence of categories $\mathcal{F}(\Theta) \longrightarrow \mathcal{F}(\Delta)$ established in [5, Section 3] is provided by the (covariant) functor $F:=\operatorname{Hom}_{A}(Y,-)$ and the standard modules of the quasi-hereditary algebra $S(A)$ are $\Delta(\lambda)=\operatorname{Hom}_{A}(Y, \Theta(\lambda))$. Since $Y$ is relative projective, $F$ is an exact functor on $\mathcal{F}(\Theta)$, sending left $A$-modules to left $S(A)$-modules.

The (indecomposable) projective $A$-modules are a subset of the (indecomposable) relative projective $A$-modules. By the above choice of the multiplicities $a_{\lambda}, A$ is isomorphic to a direct summand of $Y$, say $Y=A \oplus D$ for some left $A$-module $D$. Let $f$ be the projection from $Y$ onto $A$, and consider $f$ as an element of $S(A)$. Since $f S(A) f=f \operatorname{Hom}_{A}(Y, Y) f=\operatorname{Hom}_{A}(Y f, Y f)=\operatorname{Hom}_{A}(A, A) \simeq A$, the functor $H=f \cdot-$, the multiplication by $f$ from the left, is an exact functor from the category of left $S(A)$-modules to left $A$-modules.

Lemma 11.3. If $M \in \mathcal{F}(\Theta)$, then $H(F(M)) \simeq M$. In particular, $H(\Delta(i))=\Theta(i)$ and $H: \mathcal{F}(\Delta) \longrightarrow \mathcal{F}(\Theta)$ is an equivalence of categories.

Proof. If $M \in \mathcal{F}(\Theta)$, then:

$$
H(F(M))=f \cdot \operatorname{Hom}_{A}(Y, M)=\operatorname{Hom}_{A}(Y f, M)=\operatorname{Hom}_{A}(A, M) \simeq M .
$$

So, in particular, $f \cdot \Delta(i)=\Theta(i)$. Since $F: \mathcal{F}(\Theta) \longrightarrow \mathcal{F}(\Delta)$ is an equivalence of categories, $H: \mathcal{F}(\Delta) \longrightarrow \mathcal{F}(\Theta)$ is the inverse equivalence. 
The following statement is well-known for quasi-hereditary algebras with respect to the standard modules. Using the equivalence $\mathcal{F}(\Theta) \simeq \mathcal{F}(\Delta)$, or arguing directly from the definition of a standard system, it translates to algebras with a standard system. Without loss of generality we assume $\Lambda=\{1,2, \ldots, m\}$ with $1<2<\ldots<m$.

Lemma 11.4. Let $A$ be a cellular algebra such that the cell modules $\Theta$ indexed by $\Lambda=\{1,2, \ldots, m\}$ form a standard system. Let $M$ be an A-module which has a cell filtration. Then there exists a cell filtration

$$
M=M_{t} \supseteq M_{t-1} \supseteq \ldots \supseteq M_{1} \supseteq M_{0}=\{0\}
$$

and indices $0<i_{1}<i_{2}<\ldots<i_{m}=t$ such that for $1 \leq u \leq m$ :

$$
M_{i_{u}} / M_{i_{u-1}} \simeq \bigoplus_{x=1}^{m_{u}} \Theta(u)
$$

A module filtration $M=M_{i_{m}} \supseteq M_{i_{m-1}} \supseteq \ldots \supseteq M_{i_{1}} \supseteq M_{i_{0}}=\{0\}$ which is obtained by the process described in the above Lemma is called the cell chain of $M$. Unlike cell filtrations, the cell chain is physically unique.

The quasi-hereditary structure of $S(A)$. As above, Dlab and Ringel construct the relative projective module $Y$ which is filtered by cell modules. Let us relate the cell chain of $Y$ to the quasi-hereditary structure of $S(A)$ : Take the cell chain $Y=X_{m} \supseteq$ $X_{m-1} \supseteq \ldots \supseteq X_{1} \supseteq X_{0}=\{0\}$. An element $\alpha \in S(A)$ is a map $\alpha: Y \rightarrow Y$. Consider all maps $\alpha: Y \rightarrow Y$ with $\operatorname{im}(\alpha) \subseteq X_{1}$, the lowest cell layer of $Y$. This defines an ideal $I_{1}$ in $S(A)$. Next, consider all maps $\alpha: Y \rightarrow Y$ with $\operatorname{im}(\alpha) \subseteq X_{2}$, the two lowest cell layers of $Y$. This defines an ideal $I_{2}$ in $S(A)$. Continue this process to obtain a chain of two-sided ideals $S(A)=I_{m} \supseteq I_{m-1} \supseteq \ldots \supseteq I_{1} \supseteq I_{0}=\{0\}$. By [5] this chain is a heredity chain of $S(A)=\operatorname{End}_{A}(Y)$.

To obtain the cell chain of the left $A$-module $A$, we multiply the cell chain of $Y$ by $f$ : $A=Y f=X_{m} f \supseteq X_{m-1} f \supseteq \ldots \supseteq X_{1} f \supseteq X_{0} f=\{0\}$. Under the equivalence:

$$
F\left(X_{l} f\right)=\operatorname{Hom}_{A}\left(Y, X_{l} f\right) \simeq \operatorname{Hom}_{A}\left(Y, X_{l}\right) f=I_{l} f,
$$

as $S(A)$-A-bimodules. If $A$ is cellularly stratified then the chain of ideals which realizes $A$ as an iterated inflation, $\{0\}=J_{0} \subseteq J_{1} \subseteq \ldots \subseteq J_{n}=A$, viewed as a chain of left $A$-modules, may be refined to give the cell chain of $A$. So $J_{l}=X_{i_{l}} f$ for some $i_{l} \geq l$.

\section{Young modules And Schur algebras For Cellularly STRATified}

\section{ALGEBRAS}

Let $A$ be cellularly stratified with cell modules $\Theta(\lambda)$ for $\lambda$ in the index set $(\Lambda, \leq)$. Assume the cell modules of $A$ form a standard system. We now show that the modules $Y_{p r}(\lambda)$ defined in the previous section (based on results by Dlab and Ringel) satisfy typical properties of Young modules.

Definition 12.1. Let $A$ be cellularly stratified with cell modules $\Theta(\lambda)$ for $\lambda$ in the index set $(\Lambda, \leq)$. Assume the cell modules of $A$ form a standard system. Then the modules $Y_{p r}(\lambda)$ defined above are called Young modules of the algebra $A$, the algebra $S(A)=\operatorname{End}_{A}(Y)$ is called the Schur algebra corresponding to $A$. 
Remarks. (a) We define here Young modules which depend not only on the algebra $A$, but also on the standard system chosen.

(b) Recall that in a cellular algebra projectives - and not necessarily injectives - have a cell filtration. Instead of choosing relative projective covers $Y_{p r}$ with respect to $\mathcal{F}(\Theta)$, we could also have chosen relative injective hulls $Y_{\text {in }}$ with respect to $\mathcal{F}\left(\Theta^{*}\right)$. In this case $Y_{i n}(\lambda)=Y_{p r}(\lambda)^{*}$.

The Young modules defined here indeed satisfy the typical properties of Young modules of symmetric groups: we know already that they are indexed by the same set as the cell modules; they are indecomposable since via the equivalences $F$ or $H$ they correspond to the projective indecomposable $S(A)$-modules; they are isomorphic precisely when their labels are the same; they are filtered by cell modules with the following property translated from quasi-hereditary algebras through the equivalence $\mathcal{F}(\Theta) \simeq \mathcal{F}(\Delta)$ :

Proposition 12.2. The Young module $Y_{p r}(\lambda)$ is filtered by cell modules where the cell module $\Theta(\lambda)$ occurs precisely once and all other cell modules $\Theta(\mu)$ occurring in a cell filtration of $Y_{p r}(\lambda)$ satisfy $\mu>\lambda$.

A cellular algebra $A$ is quasi-hereditary if and only if the number of cell modules of $A$ equals the number of simple $A$-modules, see [20]; for cellular algebras in general, there are more cell modules (parameterized in the following by $\Lambda$ ) than simple or projective indecomposable modules (parameterized in the following by $\Lambda^{\text {simple }} \subseteq \Lambda$ ). In the case of a cellular algebra whose cell modules form a standard system, we determine the labels for which a Young module is a projective indecomposable module.

Proposition 12.3. Suppose that $A$ is a cellular algebra such that the cell modules form a standard system, and assume the notation as above. Then $Y_{p r}(\lambda)$ is a projective $A$ module if and only if $\lambda \in \Lambda^{\text {simple. }}$. In this case $Y_{p r}(\lambda)$ is the projective cover of the simple A-module $L_{A}(\lambda)$.

Proof. Let $S(A)$ be the Schur algebra corresponding to $A$, as constructed in Section 11. The equivalence $f \cdot-: \mathcal{F}(\Delta) \rightarrow \mathcal{F}(\Theta)$ sends indecomposable projective $S(A)$-modules to indecomposable, relative projective $A$-modules in $\mathcal{F}(\Theta)$. Write $1_{S(A)}=f+(1-f)$, and decompose both idempotents into a sum of pairwise orthogonal primitive idempotents

$$
\begin{aligned}
f & =\sum_{\mu \in \Lambda^{\text {simple }}} f_{\mu}, \\
1-f & =\sum_{\mu \in \Lambda \backslash \Lambda^{\text {simple }}} g_{\nu} .
\end{aligned}
$$

By construction of $S(A)$ in Section 11, $f_{\mu}$ and $g_{\nu}$ are inequivalent. The equivalence $f \cdot$ - sends the $S(A)$-projective module $S(A) f_{\mu}$ to the projective $A$-module $f S(A) f_{\mu}=(f S(A) f) f_{\mu}=A f_{\mu}$. And every indecomposable projective $A$-module occurs as some $A f_{\mu}$. Hence $f S(A) g_{\nu}$ cannot be projective. Moreover, $S(A) f_{\mu}$ is the projective cover of $\Delta(\mu)$ and of $L_{S(A)}(\mu)$. By exactness of the Schur functor $f \cdot-$, the module $A f_{\mu}$ is the projective cover of $f \Delta(\mu)=\Theta(\mu)$ and of $f L_{S(A)}(\mu)=L_{A}(\mu)$. Hence 
the equivalence provided by the Schur functor induces a bijection between the indecomposable projective $S(A)$-modules associated with idempotents $f_{\mu}$ and the relative projective covers $Y_{p r}(\mu)=A f_{\mu}$ of $\Theta(\mu)$, for $\mu \in \Lambda^{\text {simple }}$.

Remark. Note that we also could have used the proof of 3.7(iii) in [9] where it has been shown not only that each projective indecomposable module $P(\lambda)$ is filtered by cell modules but also that in this filtration the top quotient is the cell module $\Theta(\lambda)$.

Corollary 12.4. Let $k$ be a field of characteristic not equal to 2 or 3. Let $A$ be a cellularly stratified $k$-algebra with stratification data $\left(B_{l}, V_{l}\right)$ where each $B_{l}$ is a group algebra of a symmetric group. Choose as cell modules the dual Specht modules. Then a Young module $Y_{p r}(\lambda)$ is a projective A-module if and only if $\lambda$ is a p-restricted partition.

Proof. $Y_{p r}(\lambda)$ is a projective $A$-module if and only if there is a simple module $L_{A}(\lambda)$; this exists if and only if we have a simple module $L_{B}(\lambda)$; but $B$ is a group algebra of a symmetric group, hence the simple module $L_{B}(\lambda)$ exists if and only if $\lambda$ is $p$-restricted.

Remarks (a) If we choose as our standard system the Specht modules (instead of the dual Specht modules), then the Young module $Y_{p r}(\lambda)$ is projective if and only if $\lambda$ is a $p$-regular partition.

(b) It has been shown in [14] that the $r$-fold tensor space does not fit into a theory of Young modules for Brauer algebras, since tensor space in general does not have a cell filtration. Here we have now seen that it is possible to find a bimodule $Y$ whose direct summands are the Young modules, and whose endomorphism ring defines the Schur algebra and, as we will see in the next section, such that Schur-Weyl duality holds with respect to this bimodule.

(c) Let us examine the quasi-hereditary Schur algebra $S(A)$ in the case of Brauer algebras more closely. In this case, the definition of Young modules above agrees with that in [12] (see Definition 15 and the proof of Theorem 21 in [12]). We can write $Y=\bigoplus_{l} Y_{l}$, where $Y_{l}$ is the sum of the relative projective covers of the cell modules $G_{l}(\Theta)$, for $\Theta$ running through the cell modules of $B_{l}$ (with appropriate multiplicities). We will see that the 'diagonal subalgebra' $\operatorname{End}_{A}\left(Y_{l}\right)$ of $S(A)$ has a quotient Morita equivalent to the classical Schur algebra for the group algebra $B_{l}$ of the symmetric group. In fact, let $e=e_{l}$, and let $J^{\prime}=J_{l-1}$ be the ideal for the next lower layer, so that $B_{l} \simeq e A e / e J^{\prime} e$. Then, using the remarks preceding Proposition 14 in [12], we have

$$
0 \rightarrow J^{\prime} Y_{l} \rightarrow Y_{l} \rightarrow \widehat{Y}_{l} \otimes V_{l} \rightarrow 0,
$$

where $\widehat{Y}_{l}$ denotes the sum of the Young modules $Y^{\lambda}$ for the group algebra $B_{l}$ with appropriate multiplicities. Since these modules all have cell filtrations and $Y_{l}$ is relative projective, applying $\operatorname{Hom}_{A}\left(Y_{l},-\right)$ gives

$$
0 \rightarrow \operatorname{Hom}_{A}\left(Y_{l}, J^{\prime} Y_{l}\right) \rightarrow \operatorname{End}_{A}\left(Y_{l}\right) \rightarrow \operatorname{Hom}_{A}\left(Y_{l}, \widehat{Y}_{l} \otimes V_{l}\right) \rightarrow 0 .
$$

The application of $\operatorname{Hom}_{A}\left(-, \widehat{Y}_{l} \otimes V_{l}\right)$ to the same short exact sequence yields $\operatorname{End}_{A}\left(\widehat{Y}_{l} \otimes\right.$ $\left.V_{l}\right) \simeq \operatorname{Hom}_{A}\left(Y_{l}, \widehat{Y}_{l} \otimes V_{l}\right)$, since Proposition 8.1 implies the third term is zero. Now, 
Propositions 4.1 and 4.3 imply that $\widehat{Y}_{l} \otimes V_{l}=G_{l}\left(\widehat{Y}_{l}\right)$ and $\operatorname{End}_{A}\left(G_{l} \widehat{Y}_{l}\right) \simeq \operatorname{End}_{B_{l}}\left(\widehat{Y}_{l}\right)$. Hence we obtain that $\operatorname{End}_{B_{l}}\left(\widehat{Y}_{l}\right)$ is the quotient of $\operatorname{End}_{A}\left(Y_{l}\right)$ by $\operatorname{Hom}_{A}\left(Y_{l}, J^{\prime} Y_{l}\right)$; this quotient is Morita equivalent to the classical Schur algebra.

(d) The module $Y$ is not necessarily self-dual, unlike in the symmetric group case: When $\delta \neq 0$ if $r$ is even, then $B:=B_{\mathbb{C}}(r, \delta)$ is quasi-hereditary (see [19], Thm 3.4). Then the Young modules of $B$ are all projective indecomposable by Prop 11.3, and precisely all projective indecomposable modules occur in this way. The duals of the Young modules are then precisely all the injective indecomposable modules. This set of modules will only be the set of Young modules if the algebra is self-injective. Choose $\delta$ and $r$ (see Rui [23]) such that $B$ is not semisimple. Since, an algebra that is both quasihereditary and self-injective will be semisimple, it follows that $B$ is not self-injective. Hence some Young modules of $B$ will not be self-dual.

\section{Schur-Weyl Duality for Cellularly Stratified Algebras}

Suppose $A$ is cellularly stratified with a standard system of cell modules, indexed by a set $\Lambda$. We show that Schur-Weyl duality holds between the algebras $A$ and $S(A)$ with respect to the module $Y$.

Remark. An algebra $A$ is quasi-hereditary if and only if the algebra $A^{o p}$ is quasihereditary. In such a case the standard and costandard modules of $A$ and $A^{o p}$ are related as follows: $\Delta_{A}=\nabla_{A^{\text {op }}}^{*}$ and $\nabla_{A}=\Delta_{A^{\text {op }}}^{*}$ where ${ }^{*}$ denotes the $k$-dual of a module.

Theorem 13.1. Suppose $A$ is cellularly stratified with index set $\Lambda$ and all of the algebras $B_{l}$ have standard systems of cell modules. Then:

(1) The algebra $A$ also has a standard system of cell modules, and multiplicities in cell filtrations are well-defined.

(2) There exists a quasi-hereditary algebra $S(A)$ with the same partially ordered index set $\Lambda$ such that the following statements hold true:

(a) The category $\mathcal{F}(\Theta)$ of $A$-modules with cell filtrations is equivalent, as an exact category, to the category of $\Delta$-filtered $S(A)$-modules.

(b) The category of A-modules with cell filtrations has relative projective covers, the Young modules. The algebra $S(A)$ is the endomorphism algebra of a direct sum $Y$ of a complete set of relative projective objects in $\mathcal{F}(\Theta)$.

(c) Schur-Weyl duality holds between $A$ and $S(A)$. The faithfully balanced bimodule affording the double centralizer property between $A$ and $S(A)$ is the direct sum $Y$ of the Young modules.

Proof. The first statement is contained in Theorem 10.2 and the first two parts of the second statement have been shown in Sections 10 and 11, following [5, Section 3]. This leaves only the final part. By definition, $S(A)=\operatorname{End}_{A}(Y)$ and $Y=\bigoplus Y_{p r}(\lambda)^{a_{\lambda}}$, where $a_{\lambda}$ is chosen to equal the dimension of $L(\lambda)$ if such a simple $A$-module exists, or equals 1 otherwise, and where the sum runs through all indices $\lambda \in \Lambda$. So $Y$ is an $A$-S $(A)$-bimodule, and half of the double centralizer property holds by definition. We show the other half: Note that the projective indecomposable $A$-modules are a 
subset of the relative projective indecomposable $A$-modules. Hence $A$ is isomorphic to a direct summand of $Y$, say $Y=A \oplus D$ for some left $A$-module $D$. Let $f$ be the projection from $Y$ onto $A$, and consider $f$ as an element of $S(A)$. Clearly $f^{2}=f$, and $Y=\operatorname{Hom}_{A}(A, Y)=\operatorname{Hom}_{A}(Y f, Y)=f S(A)$ as right $S(A)$-modules; hence

$$
A=\operatorname{Hom}_{A}(A, A)=f S(A) f=\operatorname{Hom}_{S(A)}(f S(A), f S(A))=\operatorname{End}_{S(A)}(Y),
$$

and the double centralizer property holds.

Remark. We examine the connection between Schur-Weyl duality and the Dlab-Ringel equivalence in more detail. Let $A$ be a cellular algebra whose cell modules $\Theta$ form a standard system. Let $C$ be any quasi-hereditary algebra with standard modules $\Delta$ and assume that there exists an exact equivalence $\mathcal{F}(\Theta) \simeq \mathcal{F}(\Delta)$. Then the relative projective $C$-modules with respect to $\mathcal{F}(\Delta)$ are precisely the projective $C$-modules $P_{i}$, say $1 \leq i \leq t$. They correspond under the equivalence to the relative projective $A$ modules $Y_{i}$ in $\mathcal{F}(\Theta)$. Let $C=\oplus_{i=1}^{t} P_{i}^{n_{i}}$ and set $Y=\oplus_{i=1}^{t} Y_{i}^{n_{i}}$. The equivalence implies that $\operatorname{Hom}_{C}\left(P_{i}, P_{j}\right) \simeq \operatorname{Hom}_{A}\left(Y_{i}, Y_{j}\right)$ and hence

$$
C=\operatorname{End}_{C}(C) \simeq \operatorname{End}_{A}(Y) .
$$

Since $A$ is cellular and its cell modules form a standard system, all projective indecomposable $A$-modules are relative projective indecomposable modules in $\mathcal{F}(\Theta)$. Assume that these are $Y_{1}, \ldots, Y_{l}$, for some index $l \leq t$. Then $A=\oplus_{i=1}^{l} Y_{i}^{m_{i}}$ with $m_{i} \geq 1$.

Assume that $m_{i} \leq n_{i}$ for all $1 \leq i \leq l$ (otherwise one can replace $\mathrm{C}$ by a Morita equivalent algebra). Then $A$ is isomorphic to a direct summand of $Y$, say $Y=A \oplus D$ for some left $A$-module $D$. Let $f$ be the projection from $Y$ onto $A$, and consider $f \in \operatorname{End}_{A}(Y)$ as an element of $C$. Clearly $f^{2}=f$, and

$$
Y=\operatorname{Hom}_{A}(A, Y)=\operatorname{Hom}_{A}(Y f, Y)=f \operatorname{Hom}_{A}(Y, Y) \simeq f C
$$

as right $C$-modules; hence

$$
A=\operatorname{Hom}_{A}(A, A)=\operatorname{Hom}_{A}(Y f, Y f)=f C f=\operatorname{Hom}_{C}(f C, f C) \simeq \operatorname{End}_{C}(Y),
$$

and the double centralizer property holds.

\section{REFERENCES}

[1] R.Brauer, On algebras which are connected with the semisimple continuous groups. Annals of Math. 38 (1937), 854-872.

[2] E.Cline, B.Parshall, and L.Scott, Finite-dimensional algebras and highest weight categories. J. Reine Angew. Math. 391 (1988), 85-99.

[3] E.Cline, B.Parshall, and L.Scott, Stratifying endomorphism algebras. Memoir A.M.S. 124, 1996.

[4] L.Diracca and S. Koenig, Cohomological reduction by split pairs. J. Pure Appl. Algebra 212, no. 3 (2008), 471-485.

[5] V.Dlab and C.M.Ringel, The module theoretic approach to quasi-hereditary algebras. In: Representations of algebras and related topics (Kyoto, 1990), 200-224, London Math. Soc. Lecture Note Ser., 168, Cambridge Univ. Press, Cambridge, 1992.

[6] W.F.Doran And D.B.Wales, The partition algebra revisited. J. Algebra 231, no. 1 (2000), 265-330.

[7] A.Frisk, Dlab's theorem and tilting modules for stratified algebras. J. Algebra 314, no. 2 (2007), 507-537. 
[8] R.Goebel, and J.Trlifaj, Approximations and endomorphism algebras of modules. De Gruyter Expositions in Mathematics, 41. Walter de Gruyter, Berlin, 2006. xxiv +640 pp.

[9] J.J.Graham and G.I.Lehrer, Cellular algebras. Invent.Math. 123 (1996), 1-34.

[10] J.A.Green, Polynomial representations of $\mathrm{GL}_{n}$. Lecture Notes in Mathematics, 830. SpringerVerlag, Berlin-New York, 1980.

[11] D.Happel, Reduction techniques for homological conjectures. Tsukuba J. Math. 17, no. 1 (1993), $115-130$.

[12] R.Hartmann and R.Paget, Young modules and filtration multiplicities for Brauer algebras. Math.Z. 254 (2006), 333-357.

[13] D.Hemmer and D.Nakano, Specht filtrations for Hecke algebras of type A. J. London Math. Soc. (2) 69 (2004), 623-638.

[14] A.Henke and R. Paget, Brauer algebras with parameter $n=2$ acting on tensor space. To appear in Algebras and Representation Theory.

[15] G.D.JAmes Representation theory of symmetric groups. Lecture Notes in Mathematics 682, Springer, Berlin 1978.

[16] S.KoEnig, Tilting complexes, perpendicular categories and recollements of derived module categories of rings. J. Pure Appl. Alg. 73 (1991), 211-232.

[17] S.Koenig And C.C.XI, On the structure of cellular algebras. In: Algebras and modules II (Geiranger 1996), CMS Conf. Proc. 24, Amer. Math. Soc. (1998), 365-386.

[18] S.Koenig And C.C.XI, Cellular algebras: inflations and Morita equivalences. J. London Math. Soc. (2) 60 (1999), 700-722.

[19] S.Koenig and C.C.Xi Cellular algebras and quasi-hereditary algebras: a comparison. Elec. Res. Announc. Amer. Math. Soc. 5 (1999), 71-75.

[20] S.Koenig and C.C.XI, When is a cellular algebra quasi-hereditary. Math. Ann. 315 (1999), no. 2, 281-293.

[21] S.Koenig And C.C.Xi, A characteristic free approach to Brauer algebras. Trans. Amer. Math. Soc. 353 (2001), 1489-1505.

[22] P.P.Martin, Temperley-Lieb algebras for nonplanar statistical mechanics - the partition algebra construction. J. Knot Theory Ramifications 3, no. 1 (1994), 51-82.

[23] H.B.Rui, A criterion on the semisimple Brauer algebras. J. Combin. Theory Ser. A 111, no. 1 (2005), 78-88.

[24] C.C.XI, Partition algebras are cellular. Compositio Math. 119 (1999), 99-109.

[25] C.C.XI, On the quasi-heredity of Birman-Wenzl algebras. Adv. Math. 154, no. 2 (2000), 280-298.

[26] C.C.XI, On the finitistic dimension conjecture. Advances in ring theory, World Sci. Publ., (2005), 282-294.

[27] B.Zimmermann-Huisgen, Homological domino effects and the first finitistic dimension conjecture. Invent. Math. 108, no. 2 (1992), 369-383.

Anne Henke

Mathematical Institute

24-29 St Giles

University of Oxford

Oxford OX1 3LB, UK

henke@maths.ox.ac.uk
Rowena Paget

School of Mathematics, Statistics and Actuarial Science

University of Kent Canterbury CT2 7NF, UK

R.E.Paget@kent.ac.uk

Robert Hartmann, Steffen Koenig

Mathematisches Institut

Universität zu Köln

Weyertal 86-90

50931 Köln, Germany

rhartman@math.uni-koeln.de, skoenig@math.uni-koeln.de 